Document downloaded from:

http://hdl.handle.net/10251/166600

This paper must be cited as:

Sharma, S.; Garcia Tiscar, J.; Allport, JM.; Barrans, S.; Nickson, AK. (2020). Evaluation of modelling parameters for computing flow-induced noise in a small high-speed centrifugal compressor. Aerospace Science and Technology. 98:1-15.

https://doi.org/10.1016/j.ast.2020.105697

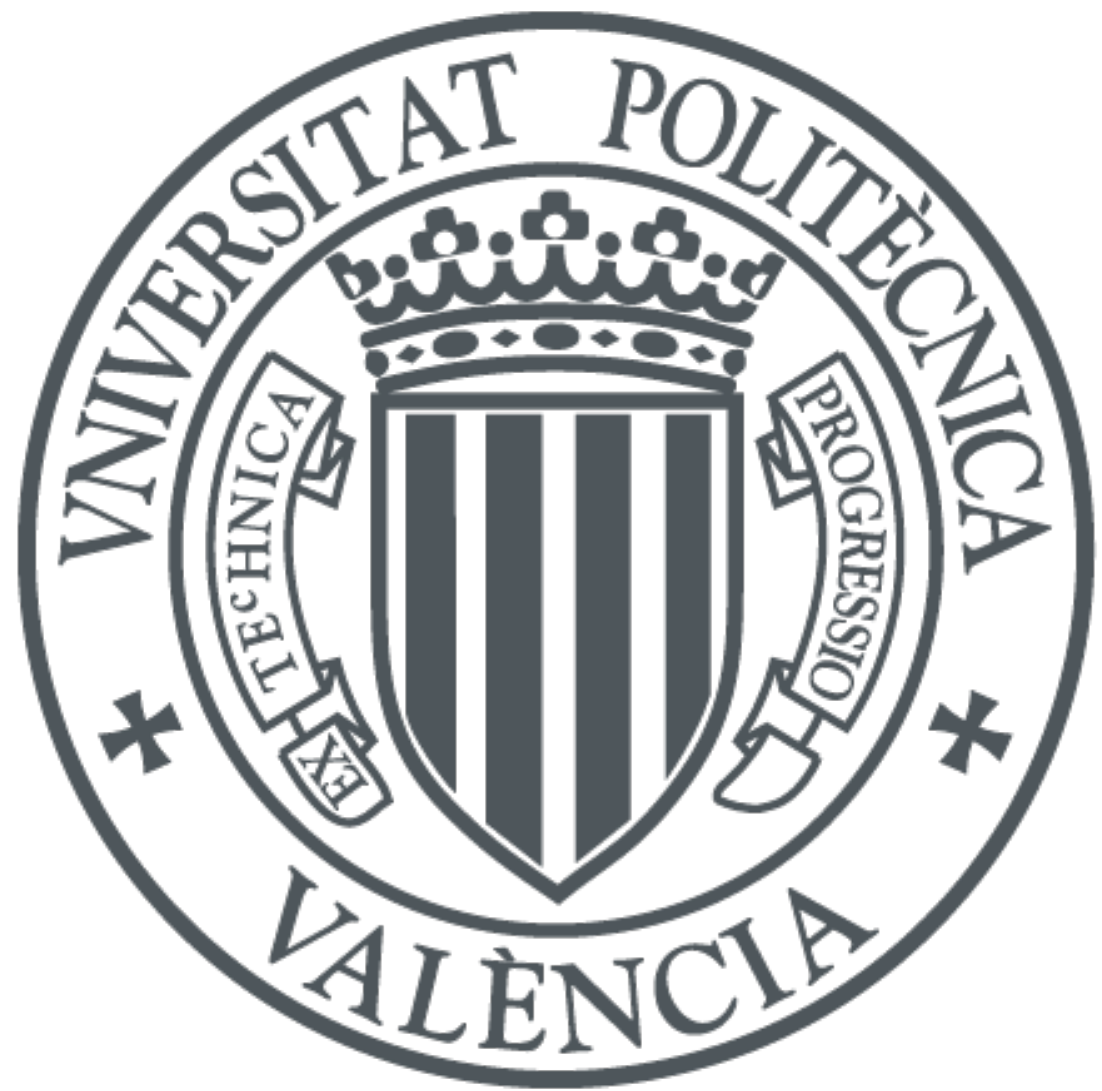

The final publication is available at

https://doi.org/10.1016/j.ast.2020.105697

Copyright Elsevier

Additional Information 


\title{
Evaluation of modelling parameters for computing flow-induced noise in a small high-speed centrifugal compressor
}

\author{
S. Sharma ${ }^{\mathrm{a}, *}$, J. García-Tíscar ${ }^{\mathrm{b}}$, J. M. Allport ${ }^{\mathrm{a}}$, S. Barrans ${ }^{\mathrm{a}}$, A. K. Nickson ${ }^{\mathrm{c}}$ \\ ${ }^{a}$ Turbocharger Research Institute, University of Huddersfield, Queensgate, Huddersfield HDI 3DH, United Kingdom \\ ${ }^{b}$ CMT - Motores Térmicos, Universitat Politècnica de València, Camino de Vera, 46022 Valencia, Spain \\ ${ }^{c}$ BorgWarner Turbo Systems Division, Bradford, West Yorkshire BD4 6SE, United Kingdom
}

\begin{abstract}
Developments in computing infrastructure and methods over the last decade have enhanced the potential of numerical methods to reasonably predict the aerodynamic noise. The generation and propagation of the flow induced noise are aerodynamic phenomena. Although the fluid flow dynamics and the resultant acoustics are both governed by mass and momentum conservation equations, former is of convective and/or diffusive nature while the latter is propagative showing insignificant attenuation due to viscosity except for small viscothermal losses. Aeroacoustic modelling of systems with intricate geometries and complex flow is still not mature due to challenges in the accurate tractable representation of turbulent viscous flows. Therefore, state-of-the-art for computing flow-induced noise in small centrifugal compressors is reviewed and critical evaluation of various parameters in the numerical model is undertaken in this work. The impact of various turbulence formulations along with corresponding spatial and temporal resolutions on performance and acoustic predictions are quantified. The performance predictions are observed to be within $1.5 \%$ of the measured values irrespective of turbulence and timestep parameters. The noise generated by the impeller is observed to be reasonably correlated with the measurements and the absolute values of the sound pressure levels along with decay rates predicted by LES and SBES formulations are better than the similar predictions from DES and URANS formulations. The impact of timestep size is observed and is determinant of the frequency up to which spectra can be appropriately resolved. Furthermore, results emphasise the importance of high spatial resolution for scale resolving turbulence formulations to yield better results and the information can be used to select appropriate numerical configuration considering time and accuracy trade-offs.
\end{abstract}

Keywords: Aeroacoustics, LES, SBES, DES, Compressor, Noise

\section{Introduction}

With the increase of applications like unmanned aerial vehicles (UAVs), auxiliary power units (APUs), turbochargers and micro gas turbines using small high-speed centrifugal compressors, the noise emission of these compressors

\footnotetext{
${ }^{*}$ Corresponding author. Tel.: +44 1484471084 ,

email: sidharath.sharma@hud.ac.uk
} 


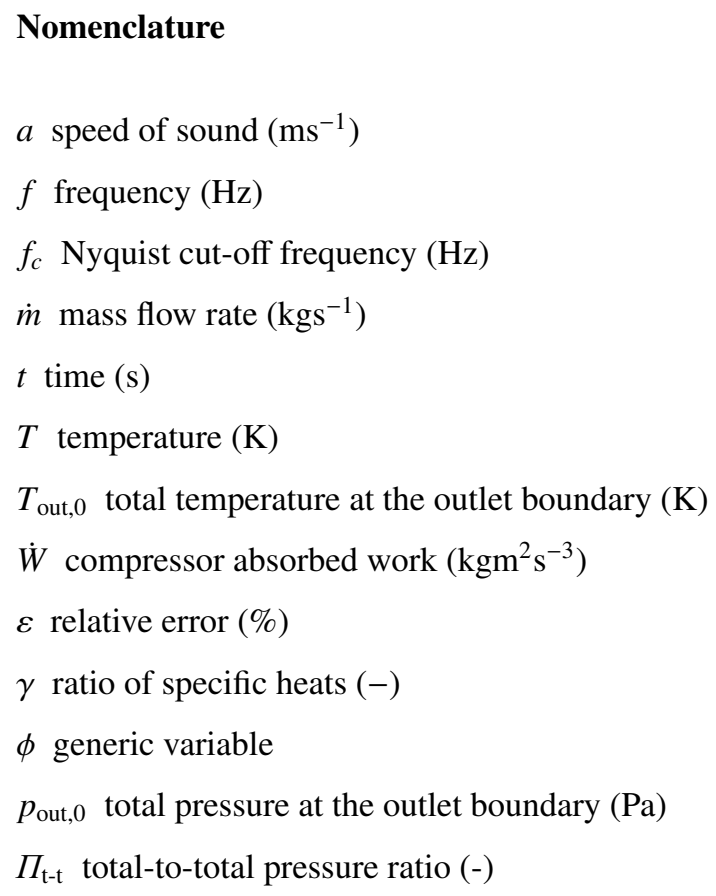

$\eta_{s}$ isentropic efficiency (-)

$y^{+}$dimensionless wall distance (-)

BPF blade passing frequency $(\mathrm{Hz})$

DES detached eddy simulation

LES large eddy simulation

PS ported shroud

PSD pressure spectral density $(\mathrm{dB})$

RANS Reynolds-averaged Navier-Stokes

$\mathrm{RBM}$ rigid body motion

$\mathrm{RO}$ rotating order

SBES stress-blended eddy simulation

SST shear-stress transport

WALE wall adopted local eddy viscosity

have become a critical aspect of product design [1]. While experimental methods can be used to characterise the acoustic behaviour of a compressor system, identification of the flow mechanisms required to understand noise generation in such complex turbomachinery systems is currently not within reach of contemporary measurement methodologies. The accurate prediction of the flow-induced noise and corresponding aerodynamic mechanisms are inherently dependent on the capability of the numerical model to accurately replicate the flow dynamics of the system. Since the direct approach of modelling flow-induced noise in small high-speed compressors is still not mature, a comprehensive evaluation of various crucial parameters in the numerical set-up of a ported shroud (PS) centrifugal compressor are undertaken in this work.

The potential of numerically computing flow-induced noise in turbomachines, specifically for the small highspeed compressor was established by the works of Mendonça et al. [2] and Karim et al. [3]. Mendonça et al. [2] computed in-duct noise using a (Delayed)Detached Eddy Simulation (DES) approach for modelling turbulence with a timestep corresponding to $1^{\circ}$ impeller rotation per iteration. Karim et al. [3] used a Large Eddy Simulation (LES) formulation for turbulence in their work. While measured spectra were not presented by Mendonça et al. [2], Karim et al. [3] demonstrated the agreement of predicted noise reduction with measured values without exactly comparing the predicted and measured value. The numerical configuration employed by Mendonça et al. [2] further stimulated the numerical studies of Broatch et al. [4, 5] and Galindo et al. [6], in which a reasonable correlation between numerical and measured acoustic spectra is demonstrated. Fontenasi et al. [7] also used DES to investigate a specific noise 
seen in the experimental measurements. Although the comparison of estimated spectra with measured spectra was not presented, specific features observed in the measurements were replicated by the numerical model.

Sundström and Mihăescu $[8,9,10]$ computed the acoustic characteristics of a turbocharger compressor using LES and explored various flow instabilities along with their corresponding mechanisms. The flow characteristics predicted by the numerical model were validated using the PIV flow measurements at a plane upstream to the impeller and a reasonable correlation between numerical and measured acoustic spectra [9] was observed. Reduced order modelling of flow variables using Dynamic Mode Decomposition (DMD) [11, 5] and Proper Orthogonal Decomposition (POD) $[12,13]$ are being actively used to investigate the flow mechanisms. Semlitsch and Mihăescu [11] and Sundström et al. [9] analysed the flow instabilities associated with the compressor operating at lower mass flow rates while Sharma et al. [12,13] explored links between high-energy flow and acoustic features using mode decomposition.

Fardafshar and Koutsovasilis [14] explored the impact of the ported shroud by modelling the near-field spectra of the compressors using the SST-SAS [15] turbulence model while Möhring's acoustic analogy [16] was used to model far-field noise propagation. The impact of the ported shroud was reasonably captured by the numerical model although the comparison between predicted and measured spectra was not good, possibly due to the inability of the formulation to resolve turbulent features. Various numerical parameters adopted in the aforementioned studies are presented in Tab. 1.

Other than the aforementioned works, the investigations of Després et al. [17], Guo et al. [18], Tomita et al. [19], Semlitsch et al. [20], JyothishKumar et al. [21], Ma et al. [22], Yang et al. [23], He et al. [24] and Shahin et al. [25] modelled the compressor using three-dimensional CFD to gain insight into the flow dynamics, specifically at stall and/or surge. Although these works are not focused on the flow-induced noise, the numerical configurations can be used to model aerodynamic noise sources.

In this paper a baseline numerical configuration is presented on the basis of the literature. The ability of this baseline configuration to yield meaningful aerodynamic and acoustic predictions is assessed by comparing numerical results with the corresponding experimental measurements. The critical decisions on the numerical set-up of the compressor which includes turbulence formulation, temporal and spatial resolution are then evaluated from the standpoint of predicting flow-induced noise with reasonable computational efficiency.

\section{Experimental measurements}

The measurements presented in this work were carried out in a flow bench facility hosted in CMT-Motores Térmicos [26] and details of the flow bench can be found in the thesis of García-Tíscar [27]. The turbocharger under investigation was installed on the flow bench and the ducts of the compressor were fitted with long straight pipes to ensure sufficient development of flow suitable for a plane wave to occur at the measurement sections. Two piezoelectric sensor arrays consisting of three piezoelectric sensors each were positioned, one each at the inlet and outlet duct measurement section. The average pressure and temperature values were measured at the compressor 


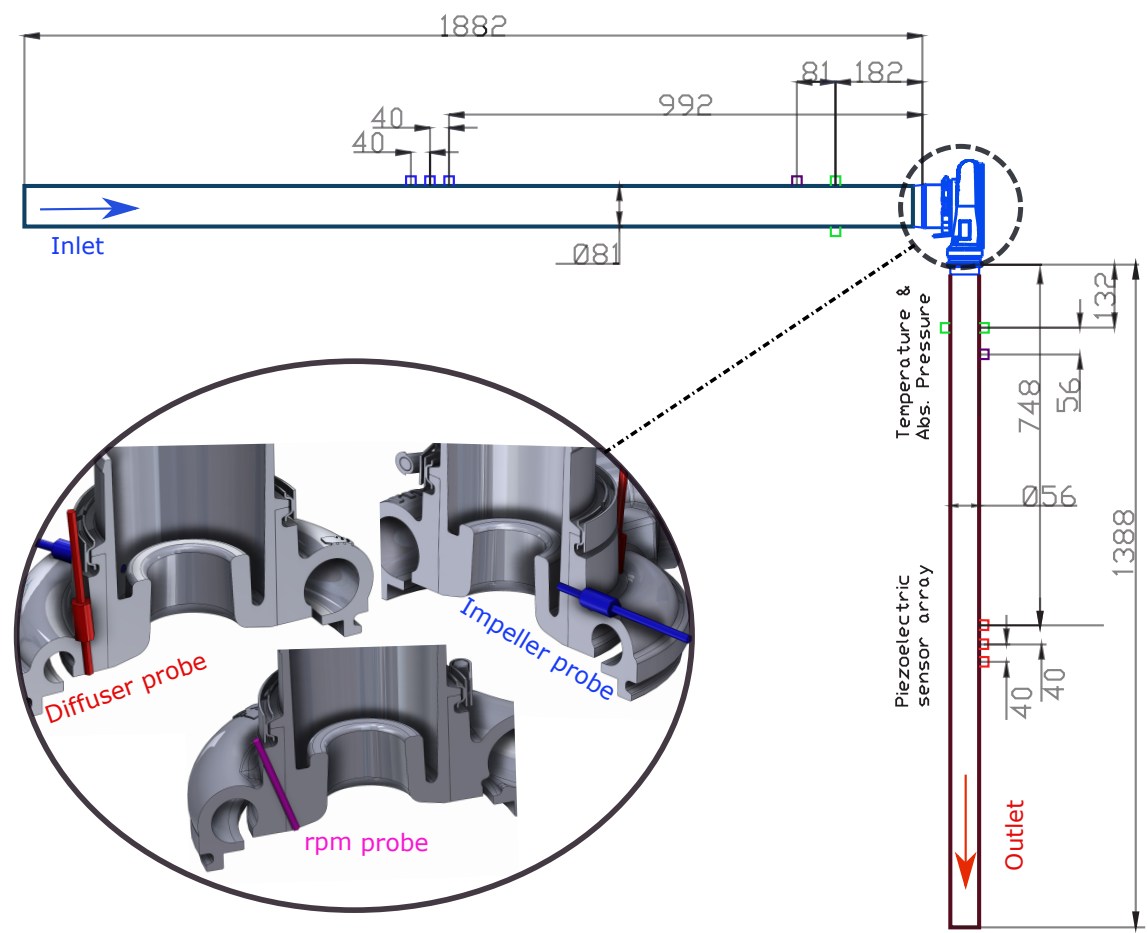

Figure 1: Schematic of the test rig used to determine the acoustic characteristics of the compressor (dimensions in mm). The location of various pressure and temperature sensors on the rig is marked. Impeller and diffuser sensor instrumented on the compressor are shown in the detailed view. The bottom figure highlights the difference in the location of experimental (wall flushed) and numerical (mid flow) probes.

inlet and outlet sections while the air mass flow rate was only measured at the compressor inlet. A schematic of the compressor measurement layout showing various sensors and their positions are shown in the Fig. 1.

The performance of the compressor was characterised by computing total-to-total pressure ratio $\Pi_{t-t}$ and isentropic efficiency $\eta_{s}$ obtained by measuring the thermodynamic variables of the operational state.

$$
\begin{aligned}
\Pi_{t-t} & =\frac{p_{\text {out }, 0}}{p_{\text {in }, 0}} \\
\eta_{s}=\frac{\dot{W}_{i s}}{\dot{W}} & =\frac{T_{\text {in }, 0}}{T_{\text {out }, 0}-T_{\text {in }, 0}}\left(\Pi_{t-t}^{\gamma-1 / \gamma}-1\right)
\end{aligned}
$$

In addition to the sensors positioned in inlet and outlet ducts, the compressor was instrumented to quantify the noise generated by the impeller using two miniaturised pressure probes positioned in the inducer and diffuser of the compressor as seen in Fig. 1. Dynamic pressure fluctuations obtained from the piezoelectric sensor arrays and miniaturised pressure probes were recorded for $1 \mathrm{~s}$ with a sampling frequency of $200 \mathrm{kHz}$. The acoustic spectra presented in terms of Power Spectral Density (PSD) are obtained using Welch's overlapped segmented averaging [28] of the measured pressure data with the number of blocks selected to achieve a frequency resolution of approximately $50 \mathrm{~Hz}$. Further details on the experimental campaign are presented in Sharma et al. [29].

It is worth pointing out while the probes used in experiments were wall flushed, the pressure in numerical model 


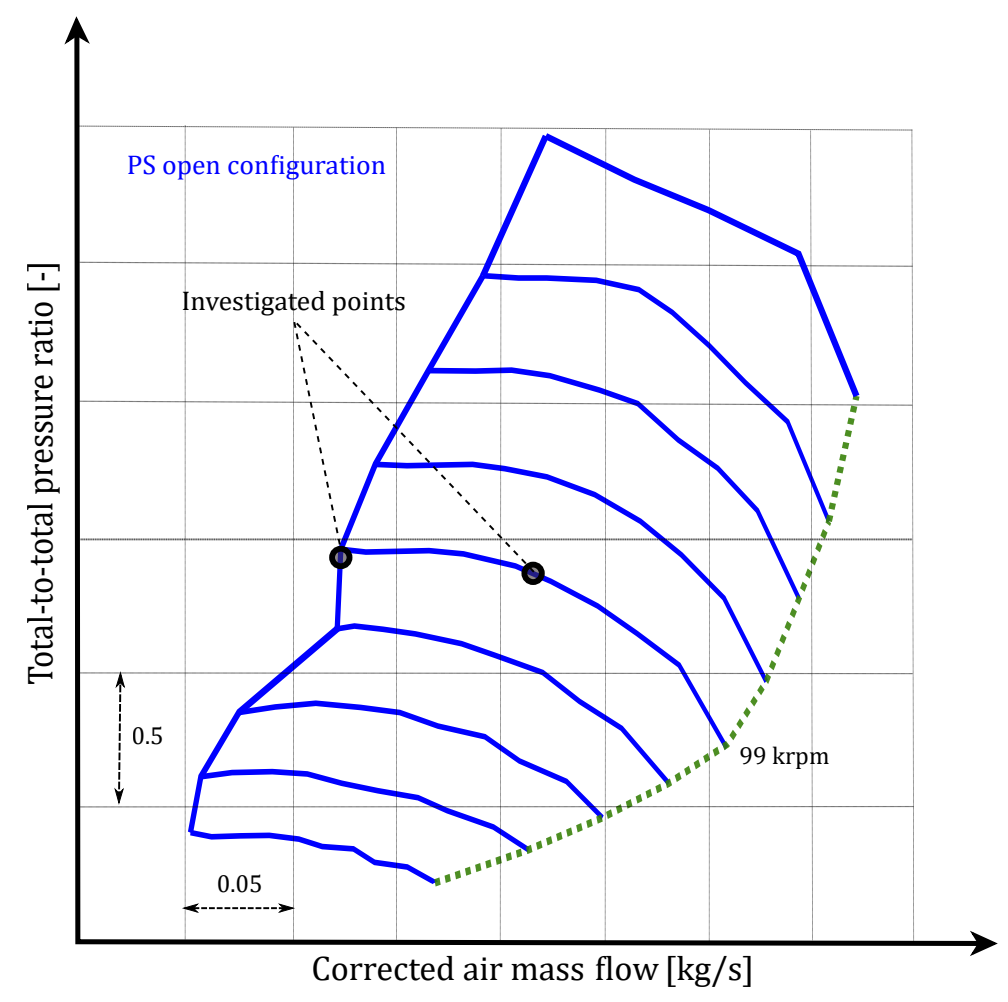

Figure 2: Compressor map marking the design and near surge conditions on which the impact of various numerical parameters are investigated.

was monitored away from the wall. The constraint of mid-flow probes in the numerical model was based on the necessity of particularly high spatial resolution to accurately model the wall-bounded flows at high Reynolds number. The boundary layer effects captured in the experimental measurements were ignored in the numerical model. In addition to that, the pressure data for various numerical configurations was recorded for $0.1 \mathrm{~s}$ ( $\approx 165$ revolutions) which includes $0.02 \mathrm{~s}$ of initial transient data.

\section{Baseline numerical configuration}

A preliminary numerical configuration was realised on the basis of work presented by Mendonça et al. [2] and Broatch et al. [4]. The various set-up parameters like grid density, timestep size, turbulence formulation and boundary conditions were carefully selected with an intent to achieve high computational efficiency, i.e. to obtain reasonable accuracy with available computational resources.

\subsection{Baseline model}

A numerical model of the compressor was built using the packages provided in ANSYS [30, 31] workbench. The digital geometry of the compressor provided by the industrial partner was cleaned up to extract the internal fluid volume. The impeller featured seven main and seven splitter blades that were designed as forward swept disseminating 


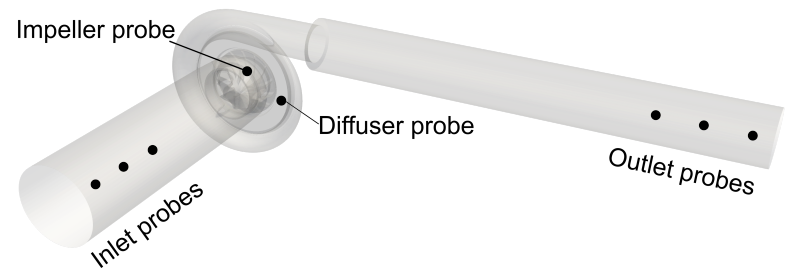

Figure 3: Computational domain along with the location of various virtual pressure probes. The duct probes are placed along the central axis.

the flow into a vaneless diffuser. The tip clearance and the impeller backside cavity were included in the model. The complete length of inlet and outlet ducts used in the measurement rig (see Fig. 1) were not included in the model because of computational intractability, although parts of these ducts equivalent to 4 cross-sectional diameters long were modelled. The intent of including these duct sections was to decrease the impact of the boundary conditions on the mean flow as well as to capture the flow instability that might propagate upstream of the impeller. The modelled domain for the compressor is shown in Fig. 3.

The computational domain was spatially discretised by an unstructured polyhedral control volume created from the tetrahedral cells generated in the ICEM CFD [30] by the vertex centred numerical approach in ANSYS CFX [31]. A domain size of approximately 10 million cells with approximately 5.5 million cells in the rotating region was adopted, taking as a reference the configuration used by Broatch et al. [4]. A polyhedral control volume is less diffusive and offers significant accuracy gains compared to an equivalent size unstructured grid of other cell types (i.e. tetrahedral) [32]. Although a structured grid would cause lower numerical diffusion and commensurately higher accuracy at the cost of significantly higher user effort, it can also lead to cells with unavoidably large aspect ratios or heavy skewness in regions of geometric complexity, resulting in unacceptable discretisation error. Furthermore, a polyhedral grid is expected to offer similar accuracy over the equivalent hexahedral grid in the cases where secondary flows are relevant [33], and therefore, off-design operating conditions can be accurately modelled using a polyhedral grid. The model along with a section view of the impeller grid is shown in Fig. 4. The flow near the wall was resolved using 12 prism or inflation layers, and the height of the first cell was chosen to be $0.0005 \mathrm{~mm}$ as to obtain $y^{+}$values closer to unity for the impeller.

In spite of the prominence of scale resolving methods as the preferred choice for modelling turbulence, the statistical Reynolds averaging approach (URANS) was selected for the baseline configuration. The limitations of Reynolds averaged approach in predicting broadband aeroacoustic sources for general unsteady flow are well established, but the limitations of the model specifically for predicting flow-induced noise in compressors are not widely documented. Furthermore, the computational efficiency of the URANS methods makes broader grid sensitivity studies tractable. The introduced Reynolds stresses are modelled using a two-equation Shear Stress Transport $(k-\omega$ SST) closure model for its ability to yield an accurate solution of detaching and swirling flows [34]. The turbulence was assumed to be isotropic with the curvature correction. The air used as working fluid in the compressor was assumed to be a perfect gas with the ideal gas law calculating the local density variation and Sutherland's law approximating the 


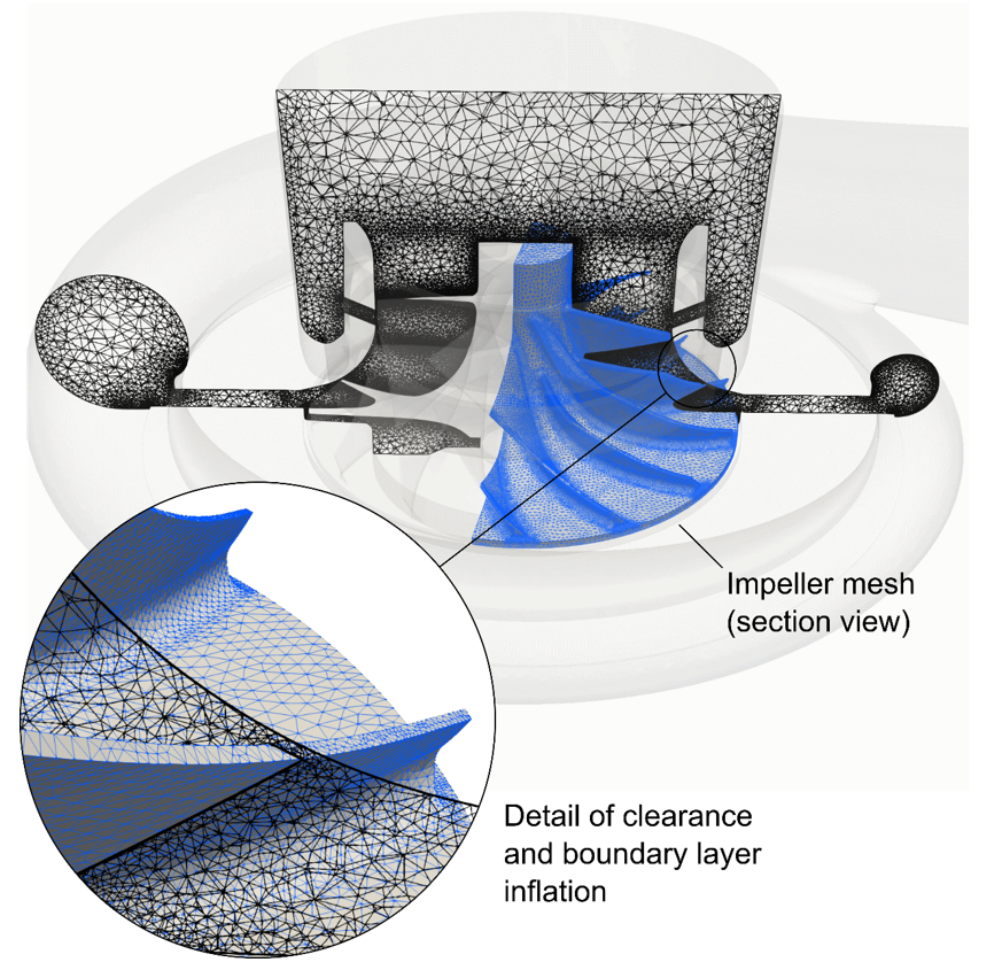

Figure 4: View of the baseline computational grid, highlighting a slice of the fluid mesh and the rotor surface mesh. A detail view shows the clearance between the blades and the shroud along with the boundary layer inflation.

dynamic viscosity.

The convective terms were discretised using a blend of second order accurate central difference scheme and firstorder upwind scheme to maintain the boundedness of the solution. The impeller motion was modelled using the rigid body sliding mesh approach. The time step for advancement was chosen in such a way that the impeller mesh turns by $4^{\circ}$ per timestep. Transient terms were discretised using an implicit, second-order accurate scheme implemented in ANSYS CFX [31] as the second order backward Euler scheme. It is worth noting that the transient scheme for the turbulence equations was still first order, and a bounded second order scheme was used for the volume fraction equations. A steady boundary condition, as a combination of the total pressure at the inlet and mass flow rate at the outlet, was used. The values of mass flow rate and pressure used as boundary conditions in the numerical model were determined from the experimental campaign. The boundary conditions for the modelled design and near surge operating points marked in Fig. 2 are presented in Tab. 2. The numerical set-up used 1\% turbulent intensity and a turbulent viscosity ratio of 10 at the inlet section. The effect of heat transfer and surface roughness was neglected by modelling wall as smooth with adiabatic and no-slip boundary conditions. Five inner coefficient loops were used for each iteration to achieve the convergence of residuals up to four orders $\left(10^{-4}\right)$. 


\subsection{Assessment of the baseline numerical configuration}

The ability of the baseline numerical configuration to yield meaningful aerodynamic and acoustic predictions was assessed by comparing numerical results with the corresponding experimental measurements.

\subsubsection{Performance parameters}

The relative differences in the pressure ratio $\Pi_{t-t}$ and isentropic efficiency $\eta_{s}$ (Eq. 1) values from experimental and numerical campaigns are presented in Tab. 3. The differences are presented in terms of relative deviation $\varepsilon$ value, which for a generic variable $\phi$, can be defined as

$$
\varepsilon(\%)=\frac{\left|\phi_{\text {num }}-\phi_{\exp }\right|}{\phi_{\exp }} 100
$$

The numerical performance parameters are slightly under-predicted, which seems counter-intuitive as the walls are modelled smooth and adiabatic. Literature [35] shows that modelling the impeller backside cavity results in the lower values of the performance variables computed in the numerical model. Nevertheless, the computed results are in close agreement as the deviation from the measured values is within the range of $\pm 1.5 \%$.

\subsubsection{Pressure spectra}

The measured pressure spectra of the inducer and diffuser positions were directly compared with the numerical spectra of the corresponding virtual probes. The pressure spectra of the near surge and design conditions are evaluated in the following subsections.

Pressure spectra of the near surge condition. The experimental pressure signals were measured for $1 \mathrm{~s}$ while numerical data was computed only up till $0.1 \mathrm{~s}$ of which $0.02 \mathrm{~s}$ is treated as initial transient. Therefore, the experimental pressure data was resampled and interpolated in accordance with the numerical data for computing PSD. The spectra computed from the original experimental pressure up till $1 \mathrm{~s}$ and the resampled pressure trace of $0.08 \mathrm{~s}$ are similar as demonstrated in Fig. 5.

In Fig. 6, the near surge pressure spectra for the inducer and diffuser probes obtained from baseline numerical configuration are compared with the experimental results. The overall amplitudes and the decay of the spectra are not well predicted by the baseline numerical model. This is expected as the complete turbulent spectrum is modelled in the URANS approach unlike LES, wherein, energy-containing range and inertial subrange is resolved. In addition to that, the broadband elevation in the inducer spectrum which is expected to be caused by the flow recirculating in the PS cavity $[13,29]$ is also not captured by the baseline numerical model. Overall trend and dominant features like blade pass frequency (BPF) are reasonably reproduced in the numerical spectra of both positions.

Pressure spectra of the design condition. The numerical and experimental spectra of inducer and diffuser probes for the design condition are compared in Fig. 7. Similar to the near surge spectra, overall amplitudes are not well captured by the numerical model. Tonal features, including the 'mid-tones' that are seen in between the two rotating order (RO) 


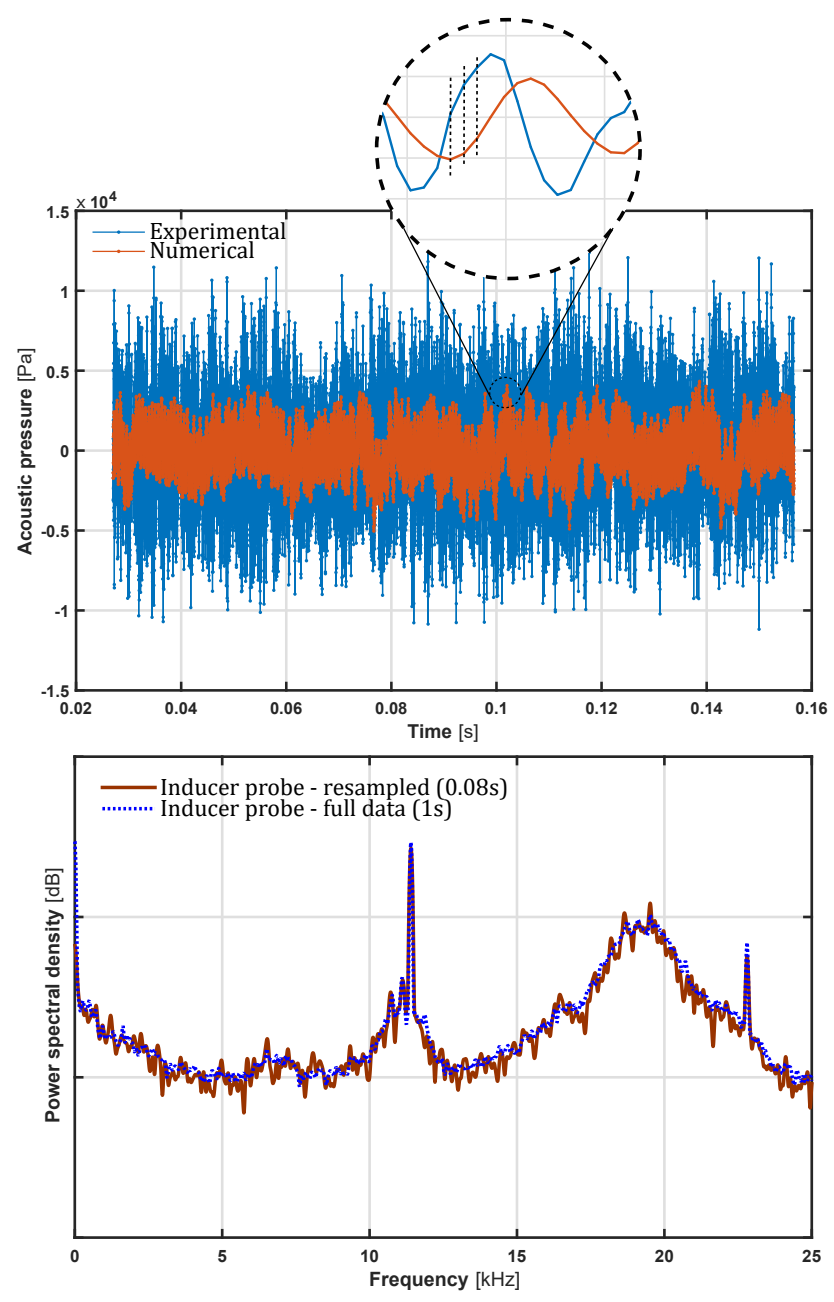

Figure 5: Numerical and interpolated values of the experimentally measured pressure for inducer probe (top). The comparison of the inducer pressure spectra obtained from measured pressure data for $1 \mathrm{~s}$ and resampled data up till $0.08 \mathrm{~s}$ (bottom).

tones, are heavily accentuated in the numerical spectra as compared to the measured spectra. Furthermore, broadband elevation in the diffuser spectra, which is expected to be caused by the interaction of diffuser outlet flow with the volute tongue $[12,29]$, is also not observed in the corresponding numerical spectra. Overall trend and dominant features like $\mathrm{BPF}$ and RO tones are reasonably captured in the numerical spectra of inducer and diffuser probes.

To summarise, the overall trends and dominant features for inducer and diffuser probes can be reasonably replicated by the baseline numerical model, but significant deviations are seen in terms of overall levels. Broadband elevation in the inducer and diffuser spectra of respective near surge and design operation is not captured by the numerical model. 

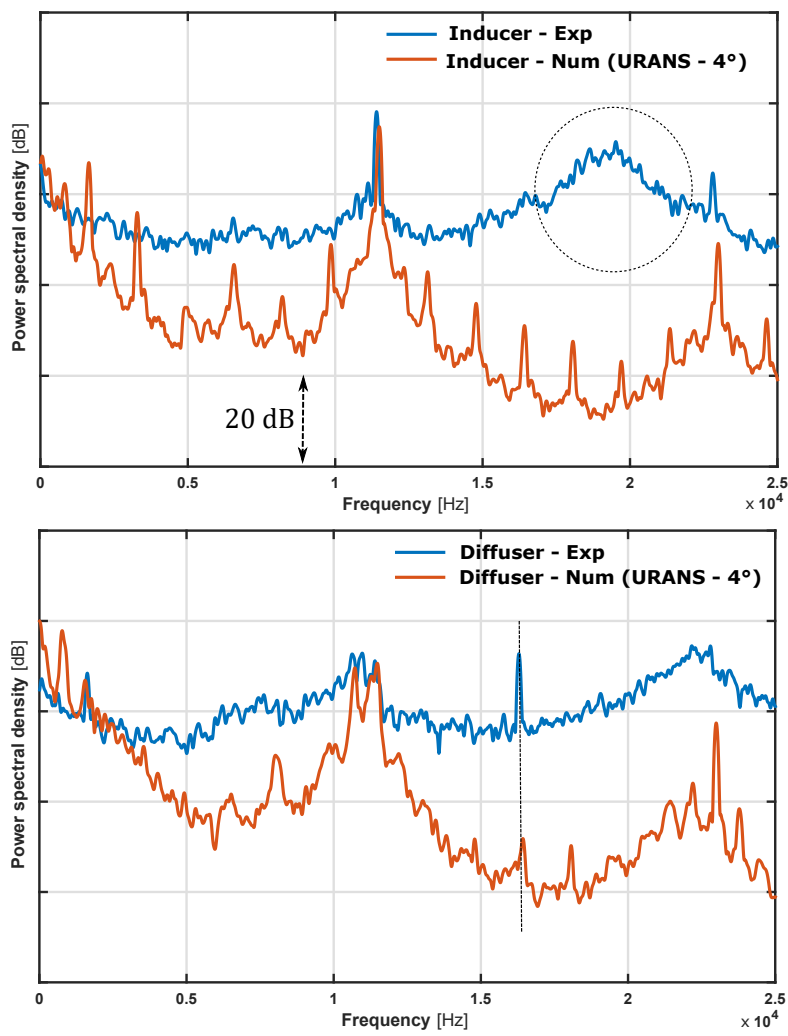

Figure 6: Comparison of the inducer (top) and diffuser (bottom) PSD predicted by the baseline numerical model with the experimentally measured values for near surge operation. The broadband feature (highlighted) in the spectrum of the inducer probe is not captured by the numerical model.

\section{Numerical modelling parameters}

In an effort to improve the credibility of acoustic predictions, various parameters and decisions on the numerical configuration are scrutinized in this section.

\subsection{Wheel rotation approach}

The primary approaches to modelling impeller motion includes Multiple Reference Frame (MRF) and Rigid Body Motion (RBM). The impeller does not actually rotate in the former approach and instead a coordinate system is used that rotates with the constant speed of wheel rotation. In the latter approach, the impeller region is actually rotated at each time step and hence, the relative position of the grids on each side of the interface is updated at each time step. With the increased accessibility of computational resources, the RBM approach is being increasingly used, and has demonstrated an improvement in the agreement between numerical and experimental data [36], especially at higher operating speeds. In addition to the RBM method, complete dynamic mesh modelling [37] can also be used to model compressor flows where the shape of the rotor domain is changing with time due to motion on the impeller walls. This model can be used to deform the boundaries in the proximity of the rotating domain but is computationally prohibitive. 

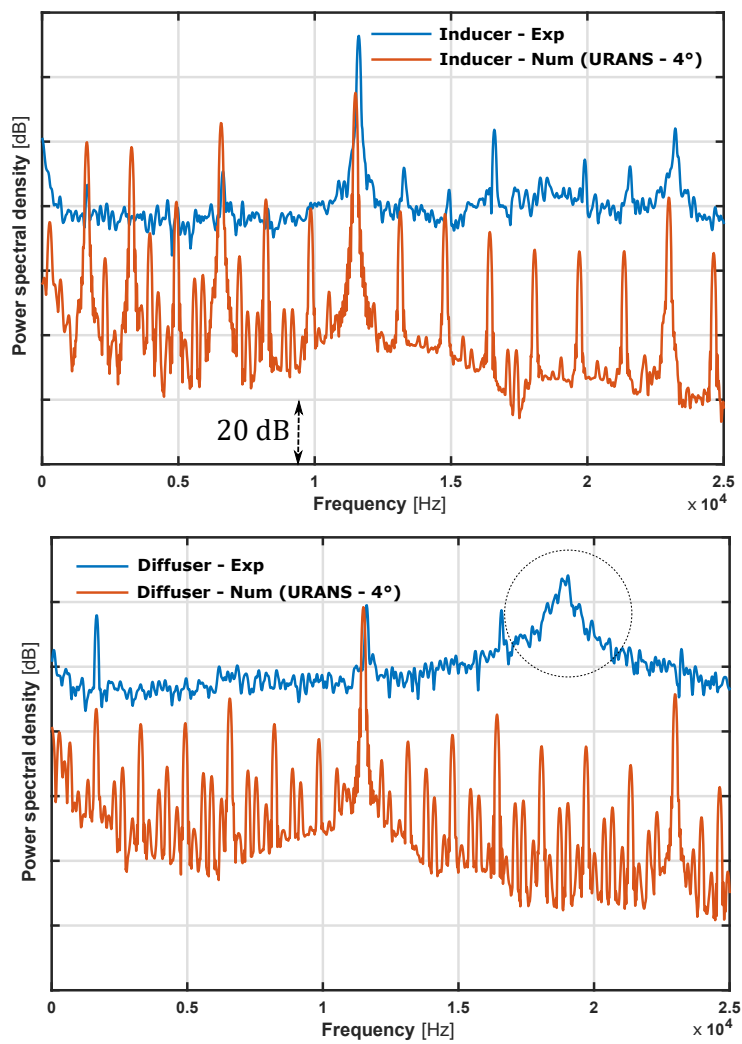

Figure 7: Comparison of the inducer (top) and diffuser (bottom) PSD predicted by the baseline numerical model with the experimentally measured values for design operation. The broadband feature (highlighted) in the spectrum of the diffuser probe is not captured by the numerical model.

The focus of this work was on understanding the acoustic features and corresponding flow mechanism in the compressor. The impact of the unsteady flow phenomenon as the sources of noise could not be neglected in this work; thereby necessitating the use of transient simulations. Although transient simulation limited to statistical turbulence models can be performed with the MRF approach, this would limit the numerical model in predicting features like $\mathrm{BPF}$ and RO tones. Therefore, transient simulations with RBM was the preferred configuration for this work.

\subsection{Boundary conditions}

The discussion is limited to the inlet and the outlet boundary conditions as the impact of heat transfer to the surrounding and surface roughness can be neglected [38] for the current problem by modelling walls using adiabatic, smooth and no-slip conditions. The boundary conditions used for modelling the compressor are primarily steady in nature. The usual sets of boundary conditions prescribed to model the centrifugal compressor as seen in the literature (see Tab. 1) include: total pressure and temperature imposed on the inlet boundary and static pressure imposed on the outlet boundary; mass flow rate on the inlet and static pressure at the outlet; total pressure and temperature on the inlet and mass flow rate at the outlet. The combination of total pressure at the inlet and static pressure at the outlet is sensitive to initial estimate [31]. Hence, the total pressure and temperature are imposed at the inlet boundary, and the 
mass flow rate is prescribed at the outlet boundary. The third of these sets of boundary conditions is identified in the ANSYS CFX modelling guide [31] as being robust and was used in this case.

\subsection{Grid or spatial resolution}

The grid of any computational model should accurately reproduce the geometry to yield credible numerical predictions. The key decisions involved in the spatial discretisation of the compressor model include determination of the type of the cells for the mesh, the number of cells and extension of the inlet and outlet ducts for maximum computational efficiency. In this section, the computational domain of the compressor is assessed to yield flow solutions independent of the mesh. This study was performed on the baseline numerical model. The global performance variables of isentropic efficiency $\eta_{s}$ and pressure ratio $\Pi_{t-t}$ (Eq. 1) were used to assess the dependence. The investigation was performed in two parts: firstly, the ability of the model to resolve the wall/boundary layer flow was evaluated for the design condition; and secondly, the minimum number of cells required in the mesh to obtain an independent solution for both design and near surge condition.

\subsubsection{Near-wall mesh resolution}

The flow near the wall is primarily defined by two parameters viz. the distance of the first cell's centroid from the wall and the thickness of the boundary layer. The near-wall region of a no-slip wall presents large gradients in the dependent variables. Also, the viscous effects on scalar transport processes like momentum transport, is large in these regions [39]. Therefore, it is necessary to accurately model the flow in the near-wall region to yield better numerical predictions of wall-bounded turbulent flows. The flow in the near-wall region is commonly modelled using two approaches, namely the wall function method and the low Reynolds (Low- $R_{e}$ ) number method. The distance of the first node from the wall is central to the application of both near-wall treatment approaches. A dimensionless wall distance parameter $y^{+}[39]$ is used to distinguish the different zones of the flow near the wall.

As mentioned before, the distance of the first cell from the wall $(y)$ was selected as $0.0005 \mathrm{~mm}$. In this section, two additional values are investigated: $(0.001 \mathrm{~mm})$ and $0.0001 \mathrm{~mm}$ yielding $y^{+}<4.5$ and $y^{+}<0.6$ respectively. The maximum value of $y^{+}$in all the cases was less than 30 implying that wall functions were not used to model the flow in the near-wall region. The viscous sublayer could be resolved by all the models as $\left(y^{+}<5\right)$. The near-wall mesh of baseline numerical configuration offers computationally and numerically optimal resolution to resolve the boundary layer as the maximum $y^{+}$was observed to be 1.6 while in the other two cases, maximum values of $y^{+}$were seen to be 0.6 and 4.5. Therefore, the baseline value of $y=0.0005 \mathrm{~mm}$ was kept as the distance of the first cell from the wall for further work.

The total thickness of the boundary layer was evaluated to ensure that it did not influence the flow solution. Prism elements, which achieve better resolution of the solution normal to the surface, were used to model the shear layer physics. The post inflation process was used to create the prism elements between the boundary shell mesh and adjacent tetrahedral elements. 12 prism layers, growing exponentially with a height ratio of 1.3 were used to model 
the boundary layer flow in the baseline configuration. Two specific height ratios of 1.2 and 1.3 ranging from 10 prism layers to 22 prism layers and 8 prism layers to 17 prism layers respectively were also explored. The global performance results of the compressor for each modelling strategy are shown in Fig. 8.

The global performance variables are seen to behave in an underdamped fashion, although the amplitude of overshoots for any performance variable is observed to be small. The baseline configuration with a height ratio of 1.3 and 12 prism layers is observed to be the computationally and numerically optimal combination for modelling boundary layer and therefore, was used in rest of this work.
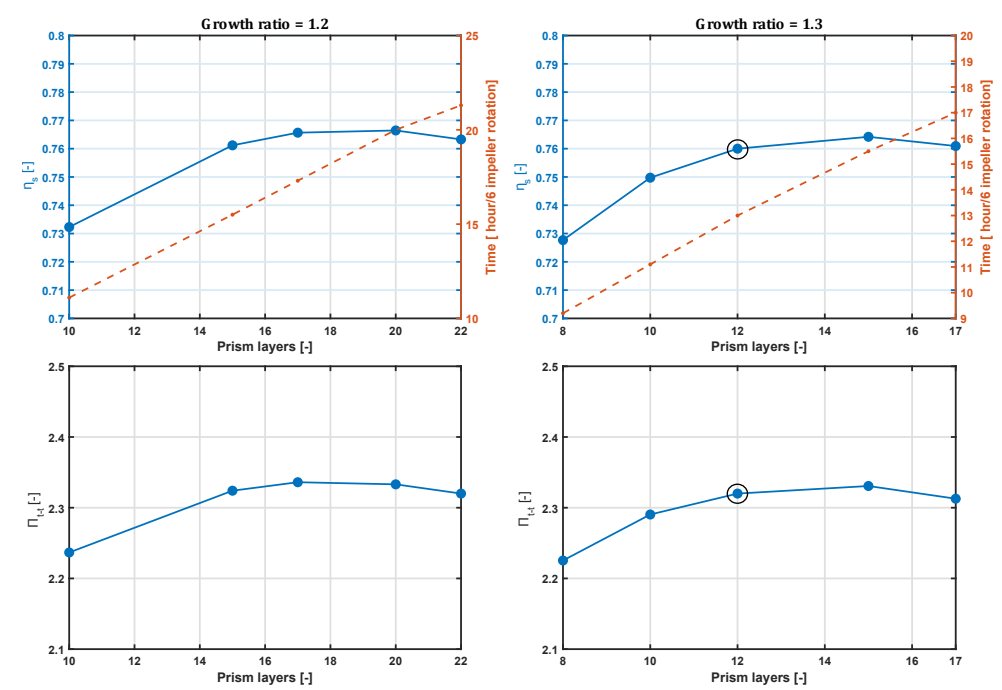

Figure 8: Global performance variables for different boundary layer parameters viz. height ratio and number of prism layers. Baseline values (marked) are proven to be optimal.

\subsubsection{Mesh resolution for free stream flow}

The sensitivity of the compressor performance variables to the free stream grid resolution was investigated by increasing the mesh density until the variation in the flow solution reached a sufficiently small value that it becomes computationally inefficient. The deviation parameter $\left(\varepsilon_{i, i+1}\right)$ was used to quantify the difference between the values of a variable in two successive cases.

$$
\varepsilon_{i, i+1}(\%)=\frac{\phi_{i+1}-\phi_{i}}{\phi_{i}} 100
$$

The results of the simulations in terms of compressor performance variables are presented in Fig. 9. The pressure ratio and isentropic efficiency for both design and near surge conditions change significantly until approximately 10 million cells, and only small fluctuations are observed for denser meshes.

The deviation $\left(\varepsilon_{i, i+1}\right)$ between the three densest meshes is less than $2 \%$ for both performance parameters at both operating conditions. Therefore, the baseline grid with 10 million cells is the computationally appropriate mesh size to model the compressor performance with reasonable accuracy. 

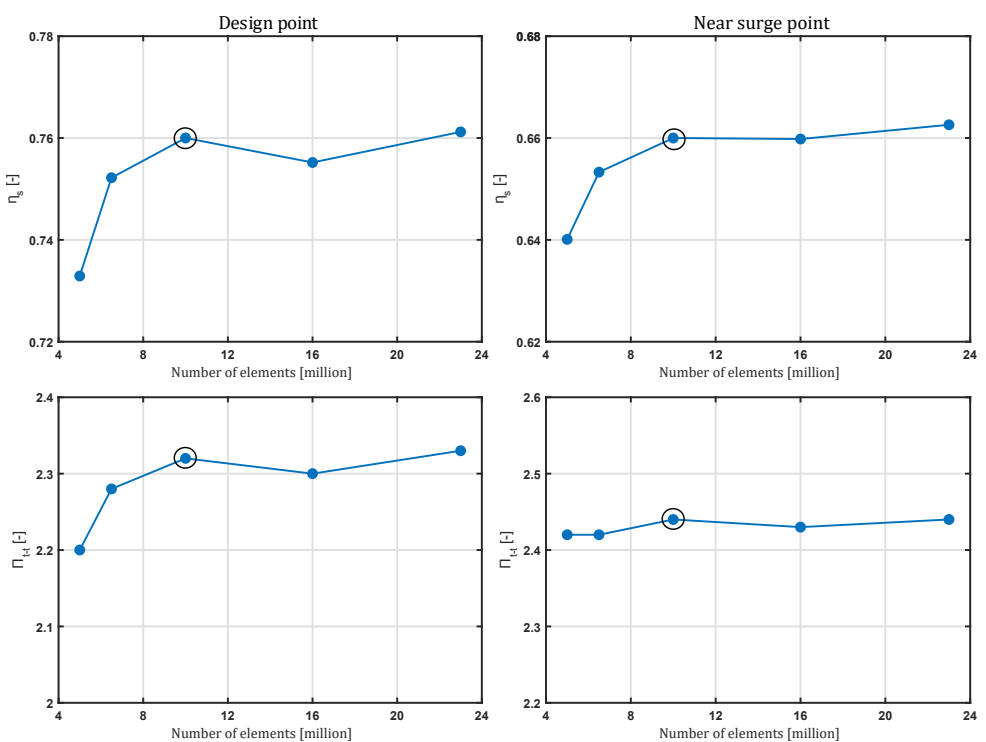

Figure 9: The sensitivity of pressure ratio and isentropic efficiency to the density of free stream grid. The grid with 10 million cells is seen as the optimal configuration.

\subsection{Turbulence formulations}

It is necessary to point out the limitation of the grid independence study described in the previous section in terms of the interpolation of the results derived on URANS onto the grids for scale resolving turbulence models. The grid requirements for the scale resolving methods are significantly higher than the URANS model, and it is not ideal to use the same grid. Similar mesh independence studies for scale resolving models, specifically LES were not computationally tractable. Therefore, the impact of grid density on performance and acoustic predictions for scale resolving turbulence model was briefly evaluated by using the finest mesh (23 million cells) as a standalone case.

Direct Numerical Simulation (DNS) resolves all the turbulent scales of interest but even with the state-of-theart computational resources, one can only handle DNS on very simple geometries at a relatively modest Reynolds number. Alternative approaches involve modelling the effects of turbulence by using an appropriate formulation without resolving all the scales of the turbulent fluctuations. Primary approaches to formulate turbulence are either statistical or scale resolving methods. While all the scales of the turbulence field are modelled in a statistical approach, scale resolving methods aim to resolve entirely or a portion of the turbulent spectrum. Large Eddy Simulation (LES) and Hybrid RANS-LES models like DES are typical scale resolving turbulence models. The recent developments in hybrid RANS-LES models include Delayed DES [40] and Stress Blended Eddy Simulation (SBES) [41].

Statistical formulation based on the Reynolds averaging of the Navier-Stokes equation (U)RANS was explored with $k-\omega$ SST [34] as the closure model. Scale resolving LES was also explored which is based on the rationale of filtering the time-dependent Navier-Stokes equation to a particular scale in the physical space. The turbulent eddies or vortices smaller than the filter scale (sub-grid scale) are modelled by appropriate means while the larger eddies 
in the flow are resolved. The use of LES to model near-wall turbulence is particularly tricky and for the flows with high Reynolds number, the computational difficulty in resolving near wall regions is further increased due to the decrease in the size of the viscous sublayer relative to the boundary layer. As most turbomachinery problems are wall-bounded flows at high Reynolds number, the LES approach would necessitate high spatial resolution. The larger scales resolved in LES aid in capturing the broadband noise sources and therefore LES is often used in computational aeroacoustics [42]. In this work, the Wall-Adopted Local Eddy-viscosity (WALE)[43] formulation was used with an intent to resolve the flow till the viscous sublayer assuming the wall resolution is sufficient. The WALE-LES uses an algebraic local eddy viscosity based subgrid model to dissipate eddies in viscous sublayer and near wall regions. This model improves upon the problem of the non-zero eddy viscosity in the laminar shear flow region observed in the Smagorinsky model [43].

The last formulation explored in this work is the combination of the above two as Hybrid RANS-LES method. In this approach, LES is employed in free shear flows and massively separated regions where turbulent structures are of a dimensionally comparable order as the geometrical structures generating them while URANS is used for the attached region and mildly separated boundary layers. The Hybrid RANS-LES models considered in this work are SBES [41] and the extended DES model based on the SST formulation proposed by Menter and Kuntz [40].

$$
\begin{aligned}
L_{t} & =\frac{\sqrt{k}}{\beta^{*} \omega} \\
L_{t, \mathrm{LES}} & =C_{\mathrm{DES}} \Delta \\
L_{t}<L_{t, \mathrm{LES}} & \equiv L_{t}<C_{\mathrm{DES}} \Delta \rightarrow \mathrm{RANS} \\
L_{t} \geq L_{t, \mathrm{LES}} & \equiv L_{t} \geq C_{\mathrm{DES}} \Delta \rightarrow \mathrm{LES} \\
\Delta & =\max \left(\Delta_{x}, \Delta_{y}, \Delta_{z}\right)
\end{aligned}
$$

Conventional DES models introduced by Spalart, Strelets and team [44] used a criterion based on the local grid size to switch between RANS and LES. The turbulent length scale $\left(L_{t}\right)$ predicted by the RANS model is compared with the equivalent length scale of the LES model $\left(L_{t}\right.$, LES $)$ based on the local grid spacing $\Delta$. The DES limiter is activated when the maximum edge length of the local computational cell is less than the turbulent length scale, implying that the turbulence length scale is larger than the local LES scale in those regions and the model switches from RANS to LES mode. The actual formulation of the conventional DES based on the two-equation model [44] implies the need for temporal and spatial resolution requirements as of the LES when grid spacing is used as the defining length scale, as all the relevant turbulence information needs to be resolved once the DES limiter is activated. Therefore, the conventional DES formulations are highly grid dependent and suffer from the issues of log-layer mismatch and grid-induced separation.

The conventional formulation of DES is extended to Delayed DES in order to 'shield' the boundary layer from the DES limiter. The reformulation of the dissipation term in the turbulent kinetic energy ( $k$-equation) for the twoequation model is compared with the one of the DES model in Eq. 5. The function $F_{\mathrm{DDES}}$ is designed to yield the 
value of $1\left(F_{\mathrm{DDES}}=1\right)$ in the boundary layer region and the value of $0\left(F_{\mathrm{DDES}}=0\right)$ for the region away from the wall [40]. The DES-SST formulation that includes the shielding properties of DDES by formulating the blending functions of the SST model as the zonal DES limiter $[40,45]$ was employed. SBES is also an improvement over conventional Detached Eddy Simulation (DES), specifically in the shielding of the boundary layer and transition issues in separating shear layers [41]. The shielding function $f_{s}$ provides improved asymptotic shielding of the RANS boundary layer against LES modification and produces significantly lower values of eddy viscosity in separating shear layers.

$$
\begin{aligned}
\varepsilon_{D E S} & =\rho \frac{k^{3 / 2}}{\min \left(L_{t}, C_{D E S}, \Delta\right)}=\rho \frac{k^{3 / 2}}{L_{t} \min \left(1, C_{D E S} \Delta_{L}\right)} \\
& =\rho \frac{k^{3 / 2}}{L_{t}} \max \left(1, \frac{L_{t}}{C_{D E S} \Delta}\right) \\
\varepsilon_{D D E S} & =\rho \frac{k^{3 / 2}}{L_{t}} \max \left[1, \frac{L_{t}}{C_{D E S} \Delta}\left(1-F_{D D E S}\right)\right]
\end{aligned}
$$

To summarise, URANS, DES, SBES and LES formulations were explored for their capability to model flowinduced noise in the compressor.

\subsection{Temporal resolution}

Definition of the appropriate timestep size or temporal resolution is vital for any unsteady transient simulation, and is of particular important to predict the flow-induced noise. The adequate value of the timestep is dictated by several numerical and empirical conditions. The numerical criterion include the Nyquist condition and the Courant-Friedrichs-Lewy (CFL) condition. The former outlines the highest frequency $\left(f_{N}\right)$ that can be resolved by a certain timestep size $(\Delta t)$ while the latter provides the information on the flow of fluid across cells for each timestep. For the $20 \mathrm{kHz}-25 \mathrm{kHz}$ range, the respective timestep size computed by Eq. 6 for the Nyquist condition should be in the range of $2.5 \times 10^{-5} s-2 \times 10^{-5} s$.

$$
f_{N}=\frac{1}{2 \Delta t}
$$

The implication of the CFL condition is summed in terms of a dimensionless number commonly referred to as Courant number $(C)$

$$
C=a \frac{\Delta t}{\Delta x}
$$

where $a$ is velocity magnitude and $\Delta x$ is the distance between the adjacent mesh cells. For the explicit linear schemes, the value of Courant number should be equal or less than 1 to maintain numerical stability. The physical interpretation of this constraint is that the propagation of the flow information should be limited to the immediate neighbour of a cell. The unsteady term throughout this work was discretised using a second-order implicit temporal scheme for better accuracy. Although an implicit temporal scheme is not constrained by Courant number for numerical stability, Spalart [46] recommends a Courant number as 1 in LES for maintaining temporal accuracy. 
The empirical suggestions in literature for selecting the appropriate timestep size for turbomachinery applications often follow a Strouhal number like approach in which the timestep size is usually expressed in degrees of impeller rotation per timestep ( $\%$ TS) or a number of timesteps per blade passing (TS/BP). The recommendation of Mendonça $[2,47]$ is to use 10 timesteps per acoustical wavelength leading to $5 \times 10^{-6} s$ while ANSYS CFX [31] recommends at least 20 timesteps per blade passing. As seen from Tab. 1, the range of $5^{\circ}$ to $1^{\circ}$ impeller rotation per timestep is pervasive in literature. Exceptions to the aforementioned range are seen in the works of Després et al. [17] and Hellstrom et al. [48] with the significantly lower values of the timestep size corresponding to $0.01^{\circ}$ and $0.19^{\circ}$ impeller rotation per timestep respectively. The use of such prohibitively small timestep sizes could be due to numerical stability issues associated with explicit temporal schemes. It is worth emphasising that the optimal size of timestep is critical as the computational overhead is doubled every time the timestep size is divided by two.

By considering the above-mentioned criteria, timestep sizes corresponding to $4^{\circ}, 2^{\circ}$ and $1^{\circ}$ were explored for the various turbulence formulations. It is worth mentioning that the coarsest timestep size $4^{\circ} / \mathrm{TS}$ corresponds to $\approx 6.7 \times 10^{-6} s$ for the investigated operating speed and which in turn corresponds to a frequency resolution of $\approx 74 \mathrm{kHz}$.

\section{Evaluation for near surge conditions}

The impact of the turbulence formulation and the corresponding timestep size on performance and acoustic predictions for near surge operation are discussed here.

\subsection{Impact on performance predictions}

Table 4 presents the compressor performance parameters (Eq. 1) viz. Pressure ratio $\Pi_{t-t}$ and isentropic efficiency $\eta_{s}$ along with the relative deviation from experimental values (Eq. 2) obtained from various turbulence formulations and timestep sizes explored to model near surge operation. The performance parameters are predicted within $1.2 \%$ of the experimental values irrespective of the turbulence formulation and timestep size. Furthermore, the time-averaged velocity distribution values in the blade passages predicted by various numerical configurations (see Fig. 10) are also similar. If the objective of the model was to predict the performance, the baseline configuration (URANS $-4^{\circ}$ ) would have been the computationally optimal choice. However, this work revolves around flow-induced noise, therefore, the impact of the turbulence formulation and timestep size on the acoustic predictions needs to be considered for finalising the optimal numerical configuration.

\subsection{Impact on acoustic predictions}

The impact of turbulence formulations and corresponding timestep size on the generation of noise are quantified with the help of inducer and diffuser probes. In the spectra of inducer and diffuser probes (see Fig. 11), the impact of timestep size seems rather small irrespective of the turbulence formulation. In addition to that, the broadband elevation centred around $19 \mathrm{kHz}$ observed in the measured inducer spectrum is not captured by any numerical configuration explored in this work. Overall trends of both inducer and diffuser spectra are reasonably captured regardless of 


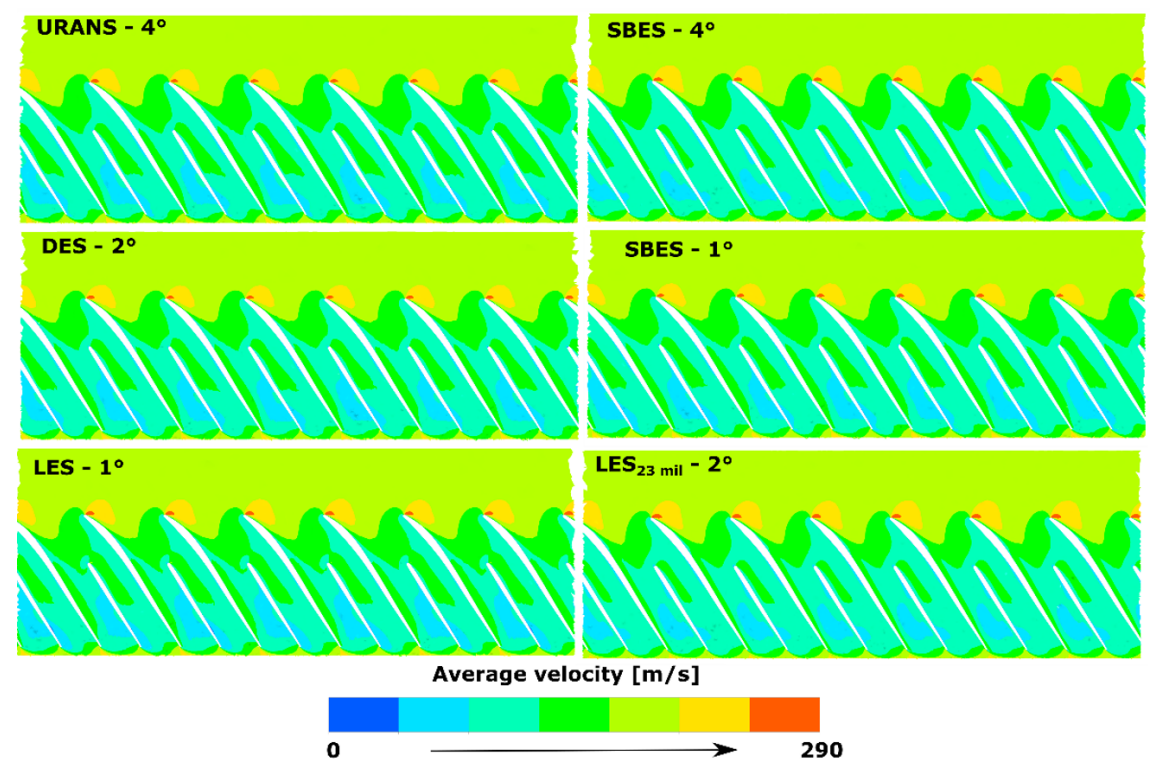

Figure 10: Average velocity distribution for the near surge point predicted by various numerical configurations at the mid impeller span.

the turbulence model. Tonal features are accentuated in the numerical spectra while overall levels are predicted significantly lower than corresponding experimental results. The correlation among overall levels is significantly improved by increasing the spatial resolution as observed from the results of the models with higher grid density.

The spectra predicted by URANS and DES formulations are similar although significant improvements in terms of amplitudes and the decay rates are observed for SBES and LES. This can be understood from the contours of the blending and shielding functions presented in Fig. 12, showing that the RANS model (blending function $=1$ ) is used over a larger region for DES while LES region (shielding function $=0$ ) is higher in the SBES model. Even though the same grid is employed for both URANS and DES formulations in this work, the use of the denser grid for the DES formulation is expected to improve the predictions. The LES formulation with respective temporal and spatial resolution corresponding to $2^{\circ}$ impeller rotation per timestep and 23 million cells is seen to yield the best agreement with the measured results. This being said the baseline URANS formulation with 10 million cells and $4^{\circ}$ timestep can reasonably capture the noise generated in the impeller operating near surge and thereby, makes a sensible choice for industrial work focused around the impact of a particular design change.

Preliminary analysis of duct spectra indicates that above a specific frequency, the spectra abruptly decay and this frequency is inversely proportional to the size of the timestep. Therefore this cut-off frequency, up to which spectra can be appropriately predicted, increases with a decrease in the timestep. The spectra predicted by a timestep corresponding to $4^{\circ}$ deviate from the respective $2^{\circ}$ and $1^{\circ}$ spectra at approximately $9 \mathrm{kHz}$ leading to poor representation of the BPF tone in the spectra of any numerical configuration with $4^{\circ}$ timestep. The deviations among the spectra corresponding to $2^{\circ}$ and $1^{\circ}$ models are observed beyond $20 \mathrm{kHz}$. It is worth pointing out that these findings on the size of timesteps are specific to the spatial resolution and may not be directly interpolated to other grids as the Courant 

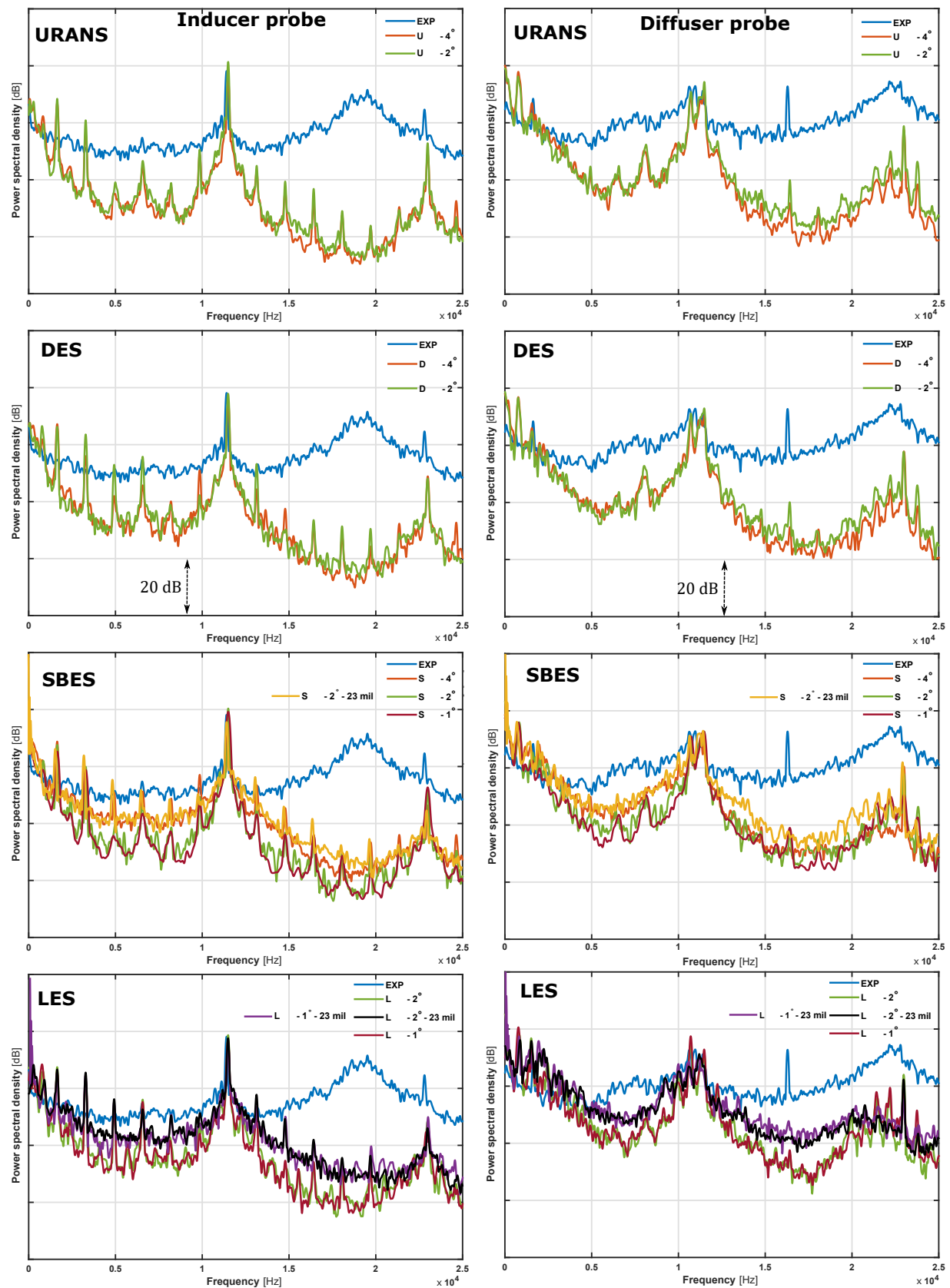

Figure 11: Inducer (left) and diffuser (right) spectra computed from various turbulence models are compared with the experimentally measured values for near surge operation.

number would change. The average Courant number in the majority of the rotor region for timesteps corresponding $1^{\circ}$ and $2^{\circ}$ is below unity (see Fig. 13), whilst a higher value of approximately two is observed in the $4^{\circ}$ timestep case. Interestingly, for the same timestep size corresponding to $2^{\circ}$, a relatively higher Courant number can be observed for the grid with the higher cell count due to the smaller element size. 


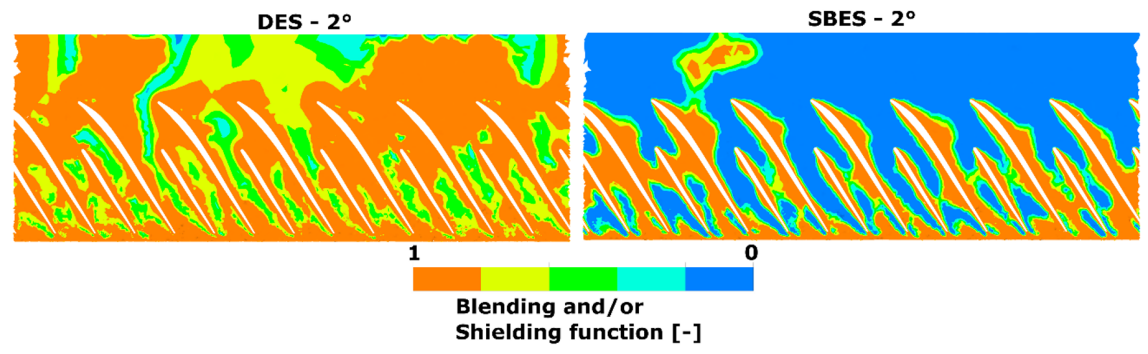

Figure 12: Blending and shielding function for the DES and SBES model respectively for the compressor operating near surge are plotted at mid-blade span. Regions with the function value as 1 are modelled using RANS while the regions with 0 indicate the use of LES model.

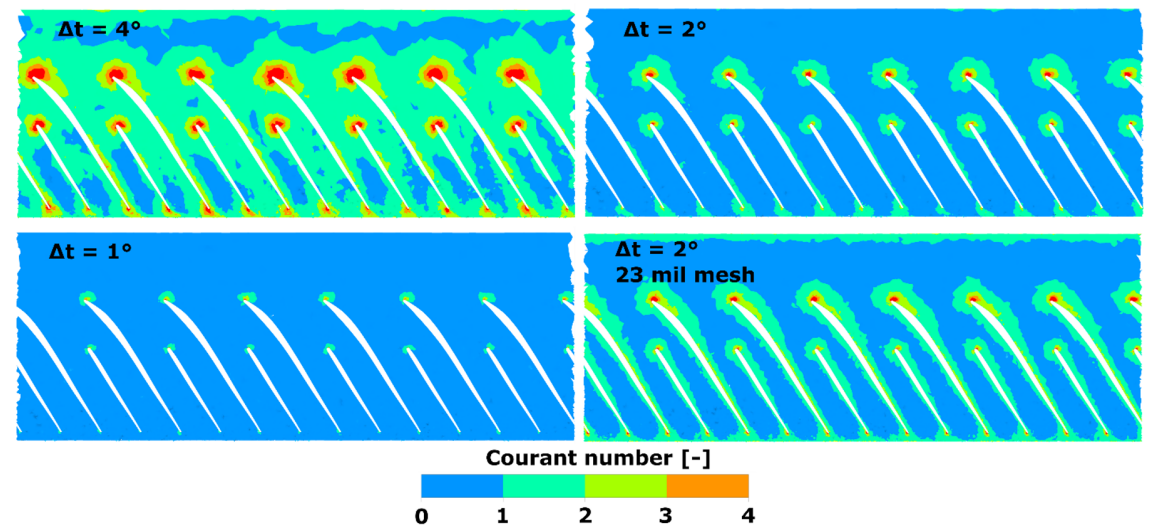

Figure 13: Contours of average Courant number in the mid-span of the impeller for different sizes of the timestep. For the same timestep size ( $\Delta t$ $=2^{\circ}$ ), an increase in spatial resolution demonstrates an increase in Courant number.
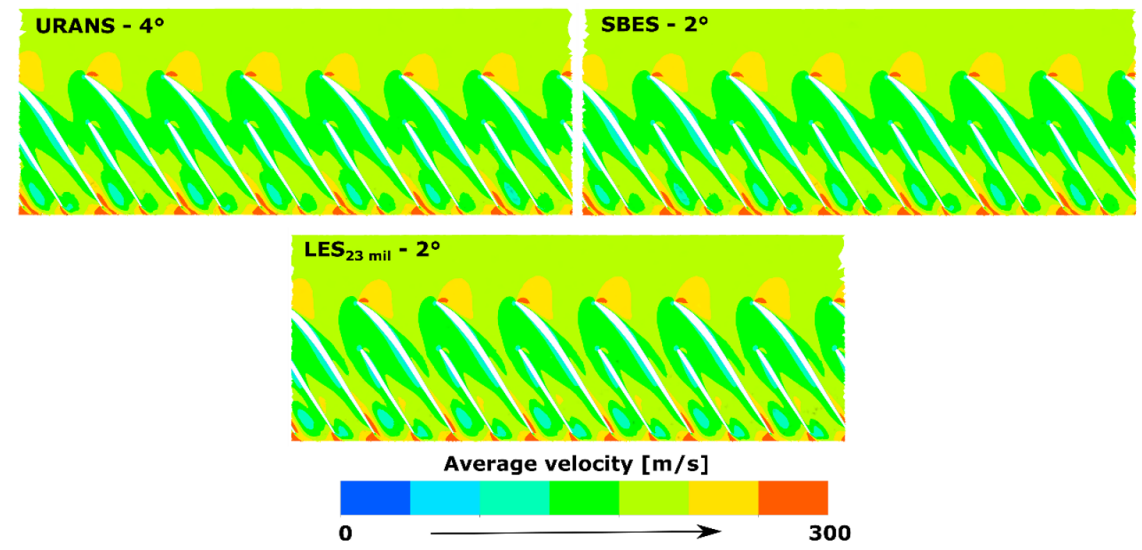

Figure 14: Average velocity distribution for the design operation predicted by various numerical configurations at the mid impeller span.

\section{Evaluation for design conditions}

Similarly, the sensitivity of performance and acoustic predictions to the turbulence formulation and timestep size for design operation are quantified in upcoming subsections. 


\subsection{Impact on performance predictions}

Compressor performance parameters and their relative deviation from experimental values for design operation are presented in Tab. 5. The performance predictions are within 1.5\% of the experimental values regardless of the turbulence formulation and timestep size. In addition to that, the velocity distributions in the impeller predicted by various models are also consistent as observed in Fig. 14. Therefore, the baseline configuration (URANS-4 ${ }^{\circ}$ ) would be the computationally optimal choice for predicting the performance characteristics of the compressor operating at design conditions too.
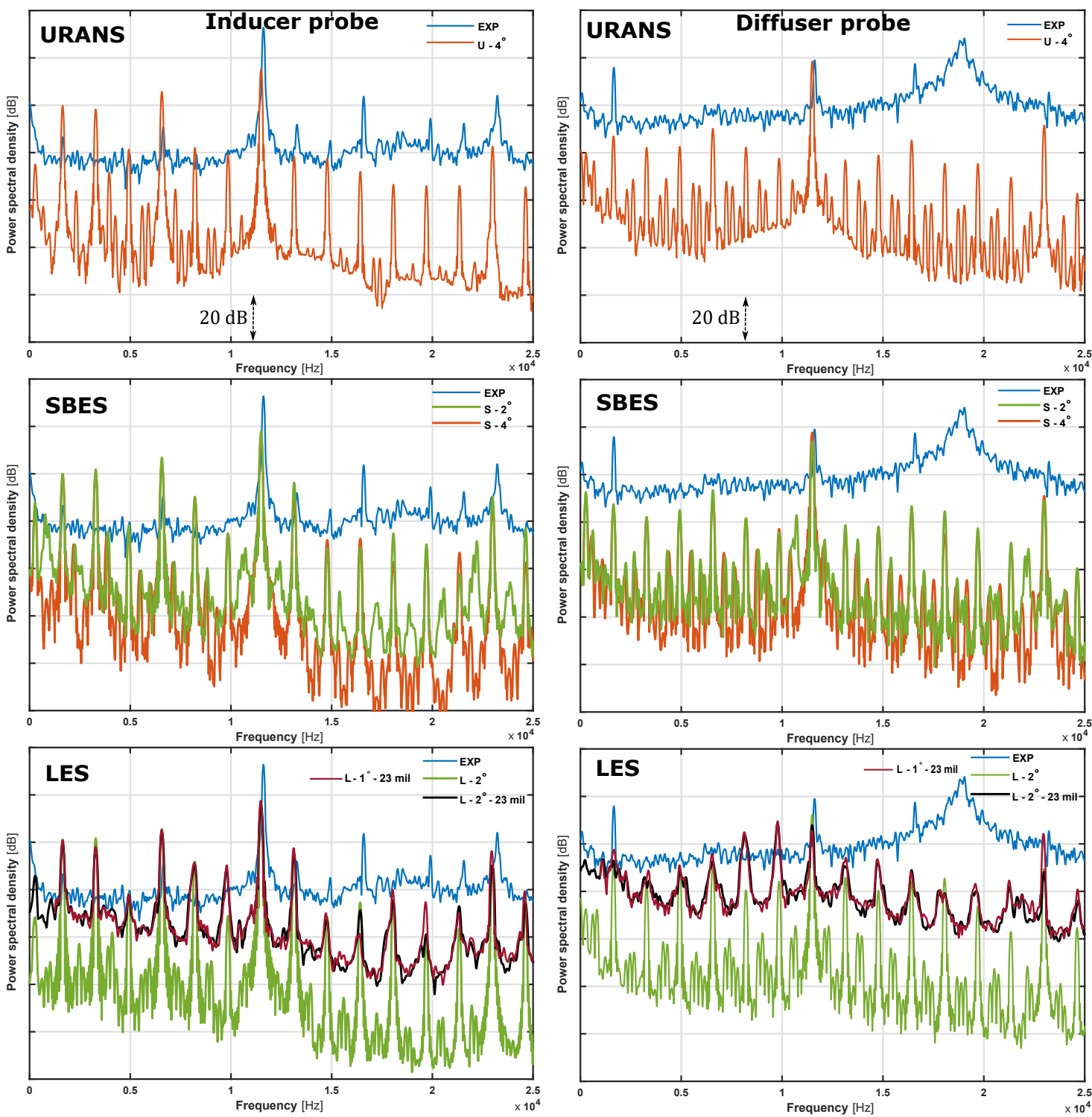

Figure 15: Inducer (left) and diffuser (right) spectra computed from various turbulence models are compared with the experimentally measured values for design operation. 


\subsection{Impact on acoustic predictions}

Similar to the near surge case, the impact of timestep size on the spectra of inducer and diffuser probes (see Fig. 15) is small irrespective of the turbulence formulation. Overall trends of both inducer and diffuser spectra are reasonably captured regardless of the turbulence formulation, although the broadband elevation observed in the region of $18.8 \mathrm{kHz}$ for the diffuser spectrum is not replicated in any numerical configuration. Tonal features are accentuated in the numerical spectra; specifically, the 'mid-tones' that are observed between adjacent RO tones are heightened as compared to experimental results. Overall levels are predicted significantly lower than corresponding experimental results for numerical models using the reference grid (10 million) while correlation is seen to be significantly improved in the LES model with higher spatial resolution (23 million). Even though the closest agreement with the measured spectra for inducer and diffuser positions is achieved by LES formulation with 23 million elements and $2^{\circ}$ timestep, the baseline numerical configuration of the URANS formulation with 10 million cells and $4^{\circ}$ timestep reasonably captures the features of the spectra and stands out as a contender on the basis of computational trade-off for industrial analysis.

To summarise, agreement with the experimental spectra for both design and near surge operating conditions is best obtained by the LES formulation with 23 million cells and $2^{\circ}$ timestep while the baseline configuration, i.e. URANS formulation with $4^{\circ}$ timestep on the grid of 10 million cells is appropriate where lower computing times are a priority. The accentuated fluctuations observed in the spectra are expected to be improved by alleviating reflections from the domain boundaries by using non-reflecting boundaries or by numerical dampening the reflections using longer ducts [5] or 'sponge zones'.

\section{Conclusions}

The development of the numerical configuration and critical evaluation of various decisions for computational modelling of flow-induced noise in a centrifugal compressor have been presented in this work. Impeller rotation was established to be modelled using an unsteady rigid body motion method, while the pressure and mass flow rate were prescribed at the inlet and outlet boundary respectively. The impact of URANS, DES, SBES and LES turbulence formulations and corresponding spatial and temporal resolutions of $4^{\circ}, 2^{\circ}$ and $1^{\circ}$ impeller rotation on performance and acoustic predictions are quantified. The results emphasise the importance of high spatial resolution for scale resolving turbulence formulations to yield better results. Furthermore, the information can be used to select appropriate numerical configuration considering time and accuracy trade-offs.

Although the baseline numerical configuration was established to be an appropriate choice in terms of spatial resolution for both boundary layer and free stream flow, a grid with double the number of elements of baseline model was seen to improve the acoustic predictions for LES formulations. The performance predictions were observed to be within $1.5 \%$ of the measured values irrespective of turbulence and timestep parameters. Furthermore, the velocity 
distribution results in the impeller as predicted by different turbulence formulations were consistent. Therefore, the baseline model can be seen as the optimal choice for performance investigations.

Although the overall trend of the spectra for the noise generation quantified from inducer and diffuser probes are similar regardless of turbulence formulations and timestep sizes, tonal features are accentuated in the numerical spectra. The absolute values of the sound pressure levels and decay rates predicted by LES and SBES formulations are better than the similar predictions from DES and URANS formulations. Further improvements in the calculation of overall levels are observed by using high spatial resolution. The broadband elevations focused around approximately $19 \mathrm{kHz}$ observed in the experimental spectra of inducer and diffuser probes for near surge and design operation respectively are not captured by any numerical configuration.

\section{Acknowledgements}

The project is sponsored and supported by BorgWarner Turbo Systems and the Regional Growth Fund (RGF Grant Award 01.09.07.01/1789C). The authors would like to thank BorgWarner Turbo Systems for permission to publish the results presented in this paper. The support of the HPC group at the University of Huddersfield is gratefully acknowledged.

\section{References}

[1] Z. Zhou, J. Huang, M. Yi, Comprehensive optimization of aerodynamic noise and radar stealth for helicopter rotor based on pareto solution, Aerospace Science and Technology 82 (2018) 607-619. doi:10.1016/j . ast.2018.09.030.

[2] F. Mendonça, O. Baris, G. Capon, Simulation of radial compressor aeroacoustics using cfd, in: Proceedings of the ASME Turbo Expo, Vol. 8, pp. 1823-1832. doi:10.1115/GT2012-70028.

[3] A. Karim, K. Miazgowicz, B. Lizotte, A. Zouani, Computational aero-acoustics simulation of compressor whoosh noise in automotive turbochargers, Tech. rep., SAE Technical Paper (2013). doi : 10.4271/2013-01-1880.

[4] A. Broatch, J. Galindo, R. Navarro, J. García-Tíscar, Methodology for experimental validation of a cfd model for predicting noise generation in centrifugal compressors, The International journal of heat and fluid flow 50 (2014) 134-144. doi:10.1016/j.ijheatfluidflow. 2014.06 .006 .

[5] A. Broatch, J. García-Tíscar, F. Roig, S. Sharma, Dynamic mode decomposition of the acoustic field in radial compressors, Aerospace Science and Technology 90 (2019) 388-400. doi:10.1016/j . ast.2019.05.015.

[6] J. Galindo, A. Tiseira, R. Navarro, M. A. López, Influence of tip clearance on flow behavior and noise generation of centrifugal compressors in near-surge conditions, International Journal of Heat and Fluid Flow 52 (0) (2015) 129-139. doi:http://dx.doi.org/10.1016/j. ijheatfluidflow.2014.12.004.

[7] S. Fontanesi, S. Paltrinieri, G. Cantore, Cfd analysis of the acoustic behavior of a centrifugal compressor for high performance engine application, Energy Procedia 45 (0) (2014) 759-768. doi:http://dx.doi.org/10.1016/j.egypro.2014.01.081.

[8] E. Sundström, B. Semlitsch, M. Mihaescu, Centrifugal Compressor: The Sound of Surge, AIAA Aviation, American Institute of Aeronautics and Astronautics, 2015. doi : 10.2514/6.2015-2674.

[9] E. Sundström, B. Semlitsch, M. Mihăescu, Generation mechanisms of rotating stall and surge in centrifugal compressors, Flow, Turbulence and Combustion 100 (3) (2018) 705-719. doi:10.1007/s10494-017-9877-z.

[10] E. Sundström, B. Semlitsch, M. Mihăescu, Acoustic signature of flow instabilities in radial compressors, Journal of Sound and Vibration 434 (2018) 221-236. doi:10.1016/j.jsv.2018.07.040. 
[11] B. Semlitsch, M. Mihăescu, Flow phenomena leading to surge in a centrifugal compressor, Energy 103 (2016) 572-587. doi:http://dx . doi.org/10.1016/j.energy.2016.03.032.

[12] S. Sharma, A. Broatch, J. Garcia-Tiscar, J. M. Allport, A. K. Nickson, Acoustic characteristics of a ported shroud turbocompressor operating at design conditions, International J of Engine Research 1 (2018) 15. doi:10.1177/1468087418814635.

[13] S. Sharma, A. Broatch, J. García-Tíscar, A. Nickson, J. Allport, Acoustic and pressure characteristics of a ported shroud turbocompressor operating at near surge conditions, Applied Acoustics 148 (2019) 434-447. doi : 10.1016/j .apacoust.2019.01.005.

[14] N. Fardafshar, P. Koutsovasilis, Ported Shroud Influence on the Aero-Acoustic Properties of Automotive Turbochargers: Quantification by Means of Simulation and Measurement, 2018.

[15] Y. Egorov, F. Menter, Development and application of sst-sas turbulence model in the desider project, in: S.-H. Peng, W. Haase (Eds.), Advances in Hybrid RANS-LES Modelling, Springer Berlin Heidelberg, pp. 261-270.

[16] W. Mohring, A well posed acoustic analogy based on a moving acoustic medium, 2010.

[17] G. Després, G. N. Boum, F. Leboeuf, D. Chalet, P. Chesse, A. Lefebvre, Simulation of near surge instabilities onset in a turbocharger compressor, Proceedings of the Institution of Mechanical Engineers, Part A: Journal of Power and Energy 227 (6) (2013) 665-673. doi: 10.1177/0957650913495537.

[18] Q. Guo, H. Chen, X. C. Zhu, Z. H. Du, Y. Zhao, Numerical simulations of stall inside a centrifugal compressor, Proceedings of the Institution of Mechanical Engineers, Part A: Journal of Power and Energy 221 (5) (2007) 683-693. doi : 10.1243/09576509JPE417.

[19] I. Tomita, S. Ibaraki, M. Furukawa, K. Yamada, The effect of tip leakage vortex for operating range enhancement of centrifugal compressor, Journal of Turbomachinery 135 (5) (2013) 051020-051020. doi : 10.1115/1.4007894.

[20] B. Semlitsch, V. Jyothishkumar, M. Mihaescu, L. Fuchs, E. Gutmark, M. Gancedo, Numerical flow analysis of a centrifugal compressor with ported and without ported shroud, SAE Technical Papers 1. doi:10.4271/2014-01-1655.

[21] V. Jyothishkumar, M. Mihai, S. Bernhard, F. Laszlo, Numerical flow analysis in centrifugal compressor near surge condition, Fluid Dynamics and Co-located Conferences, American Institute of Aeronautics and Astronautics, 2013. doi : 10.2514/6.2013-2730.

[22] S.-B. Ma, K.-Y. Kim, Optimization of discrete cavities in a centrifugal compressor to enhance operating stability, Aerospace Science and Technology 68 (2017) 308-319. doi:10.1016/j.ast.2017.05.029.

[23] C. Yang, W. Wang, H. Zhang, C. Yang, Y. Li, Investigation of stall process flow field in transonic centrifugal compressor with volute, Aerospace Science and Technology 81 (2018) 53-64. doi:10.1016/j . ast.2018.07.047.

[24] X. He, X. Zheng, Roles and mechanisms of casing treatment on different scales of flow instability in high pressure ratio centrifugal compressors, Aerospace Science and Technology 84 (2019) 734-746. doi:10.1016/j.ast.2018.10.015.

[25] I. Shahin, M. Alqaradawi, M. Gadala, O. Badr, On the aero acoustic and internal flows structure in a centrifugal compressor with hub side cavity operating at off design condition, Aerospace Science and Technology 60 (2017) 68-83. doi:10.1016/j.ast.2016.10.031.

[26] C.-M. Térmicos, Universitat politècnica de valència (1979).

URL https://www.cmt.upv.es/

[27] J. García Tíscar, Experiments on turbocharger compressor acoustics, Thesis doctoral (2017). doi:10.4995/Thesis/10251/79552.

[28] P. Welch, The use of fast fourier transform for the estimation of power spectra: A method based on time averaging over short, modified periodograms, IEEE Transactions on Audio and Electroacoustics 15 (2) (1967) 70-73. doi:10.1109/TAU.1967.1161901.

[29] S. Sharma, A. Broatch, J. García-Tíscar, J. Allport, A. Nickson, Acoustic characterisation of a small high-speed centrifugal compressor with casing treatment: An experimental study, Aerospace Science and Technologydoi:10.1016/j.ast.2019.105518.

[30] A. I. C. A. Research, ANSYS ICEM CFD Help Manual, Help System, ANSYS, Inc., USA, 2013.

[31] A. C. A. Research, ANSYS CFX-Solver Modeling Guide, Help System, ANSYS, Inc., USA, 2013.

[32] P. Chow, M. Cross, K. Pericleous, A natural extension of the conventional finite volume method into polygonal unstructured meshes for cfd application, Applied Mathematical Modelling 20 (2) (1996) 170-183. doi : http://dx.doi .org/10.1016/0307-904X (95) 00156-E.

[33] M. Tritthart, D. Gutknecht, Three-dimensional simulation of free-surface flows using polyhedral finite volumes, Engineering Applications of Computational Fluid Mechanics 1 (1) (2007) 1-14. doi : 10.1080/19942060.2007.11015177. 
[34] F. R. Menter, Two-equation eddy-viscosity turbulence models for engineering applications, AIAA Journal 32 (8) (1994) 1598-1605. doi: $10.2514 / 3.12149$.

[35] Z. Sun, C. Tan, D. Zhang, Flow field structures of the impeller backside cavity and its influences on the centrifugal compressor, Vol. 7, 2009, pp. 1349-1360. doi:10.1115/GT2009-59879.

[36] E. Sundström, B. Semlitsch, M. Mihaescu, Assessment of the $3 \mathrm{~d}$ flow in a centrifugal compressor using steady-state and unsteady flow solvers, SAE Technical Papers 2014-October. doi :10.4271/2014-01-2856.

[37] A. C. A. Research, ANSYS CFX-Solver Theory Guide, Help System, ANSYS, Inc., USA, 2013.

[38] J. Serrano, P. Olmeda, F. Arnau, M. Reyes-Belmonte, A. Lefebvre, Importance of heat transfer phenomena in small turbochargers for passenger car applications, SAE International Journal of Engines 6 (2) (2013) 716-728. doi : https ://doi .org/10.4271/2013-01-0576.

[39] S. B. Pope, Turbulent Flows, Cambridge University Press, Cambridge, 2000. doi :DOI:10.1017/CB09780511840531.

[40] F. Menter, M. Kuntz, Development and application of a zonal des turbulence model for cfx-5, Ansys, CFX-Validation Report, Technical Report No. CFX-VAL17/0503.

[41] F. Menter, Stress-blended eddy simulation (sbes)—a new paradigm in hybrid rans-les modeling, in: Symposium on Hybrid RANS-LES Methods, Springer, 2016, pp. 27-37.

[42] P. G. Tucker, Large-eddy simulation for acoustics. edited by c. wagner, t. hüttl \&amp; p. sagaut. cambridge university press, 2007.441 pp. isbn 9780521 871440. £65, Journal of Fluid Mechanics 593 (2007) 505-507. doi : 10.1017/S0022112007008725.

[43] F. Nicoud, F. Ducros, Subgrid-scale stress modelling based on the square of the velocity gradient tensor, Flow, Turbulence and Combustion 62 (3) (1999) 183-200. doi:10.1023/A:1009995426001.

[44] M. Strelets, Detached eddy simulation of massively separated flows, Aerospace Sciences Meetings, American Institute of Aeronautics and Astronautics, 2001. doi:doi:10.2514/6.2001-879.

[45] F. R. Menter, M. Kuntz, Adaptation of Eddy-Viscosity Turbulence Models to Unsteady Separated Flow Behind Vehicles, Springer Berlin Heidelberg, Berlin, Heidelberg, 2004, pp. 339-352. doi : 10.1007/978-3-540-44419-0_30.

[46] P. Spalart, C. Streett, Young-Person's Guide to Detached-Eddy Simulation Grids, 2001.

[47] F. Mendonca, A. Read, F. Imada, V. Girardi, Efficient cfd simulation process for aeroacoustic driven design, in: SAE Technical Paper 201036-0545, 2010. doi:10.4271/2010-36-0545.

[48] F. Hellstrom, E. Gutmark, L. Fuchs, Large eddy simulation of the unsteady flow in a radial compressor operating near surge, Journal of Turbomachinery 134 (5) (2012) 051006-051006. doi : 10.1115/1.4003816. 
Table 1: Literature survey of the various numerical parameters crucial to model aerodynamics and noise generation in the compressors.

\begin{tabular}{|c|c|c|c|c|c|c|c|c|c|}
\hline \multirow{2}{*}{ Study } & \multirow{2}{*}{$\begin{array}{c}\text { Tip } \\
\text { diameter } \\
{[\mathrm{mm}]}\end{array}$} & \multirow{2}{*}{$\begin{array}{l}\text { Elements } \\
\text { [million] }\end{array}$} & \multirow{2}{*}{$\begin{array}{l}\text { Prism } \\
\text { layers } \\
{[-]}\end{array}$} & \multirow{2}{*}{$\begin{array}{c}\text { Wheel } \\
\text { rotation } \\
\text { [-] }\end{array}$} & \multirow{2}{*}{$\begin{array}{c}\text { Turbulence } \\
\text { method } \\
{[-]}\end{array}$} & \multicolumn{2}{|c|}{ Boundary conditions } & \multicolumn{2}{|c|}{ Timestep size } \\
\hline & & & & & & Inlet & Outlet & ${ }^{\circ} / \mathrm{TS}$ & TS/BP \\
\hline $\begin{array}{l}\text { Fardafshar } \\
\text { et al. [14] }\end{array}$ & - & - & - & static & SAS-SST & - & - & - & - \\
\hline $\begin{array}{l}\text { Sundström } \\
\text { et al. [8] }\end{array}$ & 88 & 9 & 10 & sliding & LES & pressure & mass flow & 1 & 36 \\
\hline $\begin{array}{l}\text { Fontenasi } \\
\text { et al. [7] }\end{array}$ & - & 9.6 & 11 & sliding & DES & Mass flow & pressure & 0.5 & 120 \\
\hline $\begin{array}{l}\text { Broatch et } \\
\text { al. [4] }\end{array}$ & 48.6 & 9.6 & - & sliding & DES & Mass flow & pressure & 1 & 60 \\
\hline $\begin{array}{l}\text { Mendonça } \\
\text { et al. [2] }\end{array}$ & - & 9 & 10 & sliding & DES & pressure & pressure & 1 & 60 \\
\hline $\begin{array}{l}\text { Karim et } \\
\text { al. [3] }\end{array}$ & - & - & - & sliding & LES & pressure & mass flow & - & - \\
\hline $\begin{array}{l}\text { Semlitsch } \\
\text { et al. [20] }\end{array}$ & 88 & 6 & & sliding & LES & mass flow & pressure & 5 & 7.2 \\
\hline $\begin{array}{l}\text { JyothishKumar } \\
\text { et al. [21] }\end{array}$ & ar 88 & 6 & & sliding & LES & mass flow & pressure & 5 & 7.2 \\
\hline $\begin{array}{l}\text { Tomita et } \\
\text { al. [19] }\end{array}$ & 50 & 3.2 & & & $\begin{array}{l}\text { URANS } \\
(k-\epsilon)\end{array}$ & & & $3.6 / 7.2$ & $20 / 12.5$ \\
\hline $\begin{array}{l}\text { Guo et al. } \\
{[18]}\end{array}$ & 182.8 & 2.5 & & sliding & $\begin{array}{l}\text { URANS } \\
(k-\epsilon)\end{array}$ & pressure & mass flow & 3 & 20 \\
\hline
\end{tabular}

Table 2: Boundary conditions

\begin{tabular}{lccc} 
Case & $n[\mathrm{rpm}]$ & $p_{\text {in }, 0}[\mathrm{bar}]$ & $\dot{m}_{\text {out }}[\mathrm{kg} / \mathrm{s}]$ \\
\cline { 1 - 3 } Design & & 1 & 0.211 \\
\cline { 1 - 3 } Near surge & 98529 & 1 & 0.122 \\
\hline
\end{tabular}


Table 3: Comparison of the performance parameters predicted by baseline numerical model with the experimental measurements for design and near surge points

\begin{tabular}{lllccc}
\hline \multirow{2}{*}{ Method } & Case & $\Pi_{t-t}[-]$ & $\eta_{s}[\%]$ & $\varepsilon_{\pi}[\%]$ & $\varepsilon_{\eta}[\%]$ \\
\hline \multirow{2}{*}{ Exp } & Design & 2.35 & 76.8 & - & - \\
\cline { 2 - 6 } & Near surge & 2.47 & 66.7 & - & - \\
\hline \multirow{2}{*}{ Num } & Design & 2.32 & 76 & 1.1 & 1.1 \\
\cline { 2 - 6 } & Near surge & 2.44 & 66 & 1.2 & 1.0 \\
\hline
\end{tabular}

Table 4: Performance variables $\Pi_{t-t}$ and $\eta_{s}$ predicted by various numerical configurations for near surge operation are compared with the experimental results.

\begin{tabular}{|c|c|c|c|c|c|c|}
\hline Method & $\Delta t\left[{ }^{\circ} / \mathrm{TS}\right]$ & Grid [mil] & $\Pi_{t-t}[-]$ & $\eta_{s}[\%]$ & $\varepsilon_{\pi}[\%]$ & $\varepsilon_{\eta}[\%]$ \\
\hline Experimental & - & - & 2.47 & 66.7 & - & - \\
\hline \multirow{2}{*}{ URANS } & 4 & \multirow{2}{*}{10} & 2.44 & 66 & 1.2 & 1 \\
\hline & 2 & & 2.44 & 65.9 & 1.2 & 1.2 \\
\hline \multirow{2}{*}{ DES } & 4 & \multirow{2}{*}{10} & 2.46 & 66.1 & 0.4 & 0.9 \\
\hline & 2 & & 2.44 & 66 & 1.2 & 1 \\
\hline \multirow{3}{*}{ SBES } & 4 & \multirow{3}{*}{10} & 2.47 & 66.4 & 0.1 & 0.5 \\
\hline & 2 & & 2.45 & 66.2 & 0.8 & 0.7 \\
\hline & 1 & & 2.44 & 65.9 & 1.2 & 1.2 \\
\hline SBES & 2 & 23 & 2.47 & 66.3 & 0.1 & 0.6 \\
\hline \multirow{2}{*}{ LES } & 2 & \multirow{2}{*}{10} & 2.45 & 66.3 & 0.8 & 0.6 \\
\hline & 1 & & 2.44 & 65.7 & 1.5 & 1.2 \\
\hline \multirow[b]{2}{*}{ LES } & 2 & \multirow[b]{2}{*}{23} & 2.48 & 66.5 & 0.2 & 0.4 \\
\hline & 1 & & 2.48 & 66.5 & 0.2 & 0.4 \\
\hline
\end{tabular}


Table 5: Performance variables $\Pi_{t-t}$ and $\eta_{s}$ predicted by various numerical configurations for design point are compared with the experimental results.

\begin{tabular}{lcccccc}
\hline Method & $\Delta t[\%$ TS] & Grid [mil] & $\Pi_{t-t}[-]$ & $\eta_{s}[\%]$ & $\varepsilon_{\pi}[\%]$ & $\varepsilon_{\eta}[\%]$ \\
\hline Experimental & - & - & 2.35 & 76.8 & - \\
\hline URANS & 4 & 10 & 2.32 & 76 & 1.1 & 1.1 \\
\hline \multirow{2}{*}{ SBES } & 4 & 10 & 2.32 & 76.1 & 1 & 0.9 \\
\cline { 2 - 6 } LES & 2 & 10 & 2.33 & 76.1 & 0.9 \\
\hline \multirow{2}{*}{ LES } & 2 & & 2.37 & 78 & 1.5 \\
\hline
\end{tabular}

